\title{
Temporal Encoding: Relative and Absolute Representations of Time Guide Behavior
}

\author{
Başak Akdoğan ${ }^{1,2}$, Amita Wanar ${ }^{3}$, Benjamin K. Gersten ${ }^{1}$, Charles R. Gallistel ${ }^{4}$, and Peter D \\ Balsam $^{1,2,3}$ \\ ${ }^{1}$ Department of Psychology, Columbia University \\ ${ }^{2}$ New York State Psychiatric Institute \\ ${ }^{3}$ Department of Psychology, Barnard College \\ ${ }^{4}$ Rutgers Center for Cognitive Science, Rutgers University
}

\begin{abstract}
Author Note
The authors thank Kenneth Light and David Freestone for their constructive comments, and Brian Cotten for helping with data collection. This study was supported by National Institute of Mental Health Grant R01MH068073 to PB.

We have no known conflict of interest to disclose. The experiments reported in this article were not formally preregistered. Requests for the data or materials can be sent via email to the corresponding author.

Correspondence regarding this article should be addressed to Başak Akdoğan, Department of Psychology, Columbia University, New York, NY 10027, USA. Email: basak.akdogan@columbia.edu
\end{abstract}




\begin{abstract}
Temporal information-processing is critical for adaptive behavior and goal-directed action. It is thus crucial to understand how the temporal distance between behaviorally relevant events is encoded to guide behavior. However, research on temporal representations has yielded mixed findings as to whether organisms utilize relative versus absolute judgments of time intervals. To address this fundamental question about the timing mechanism, we tested mice in a duration discrimination procedure in which they learned to correctly categorize tones of different durations as short or long. After being trained on a pair of target intervals the mice transferred to conditions in which cue durations and corresponding response locations were systematically manipulated so that either the relative or absolute mapping remained constant. The findings indicate that transfer occurred most readily when relative relationships of durations and response locations were preserved. In contrast, when subjects had to re-map these relative relations, even when positive transfer initially occurred based on absolute mappings, their temporal discrimination performance was impaired, and they required extensive training to re-establish temporal control. These results demonstrate that mice can represent experienced durations both as having a certain magnitude (absolute representation) and as being shorter or longer of the two durations (an ordinal relation to other cue durations), with relational control having a more enduring influence in temporal discriminations.
\end{abstract}

Keywords: temporal representation, relative versus absolute, duration discrimination, transfer, time perception 


\section{Temporal Encoding: Relative and absolute representations of time guide behavior}

Temporal information-processing is critical for adaptive behavior and goal-directed

action. Our ability to assess if we should hit the brakes as traffic light turns yellow or whether the food order we placed online is running late indicates that we are able to process the passage of time and utilize this information to guide our decisions. Despite the great extent of experimental and theoretical approaches examining the psychophysical characteristics of timing behavior across species, there is not yet a consensus about how organisms represent durations. Due to the presumed similarities in the perception of time, space, and quantity (e.g., Meck \& Church, 1983; Walsh, 2003), it might well be the case that time is represented in a similar way to the spatial and numerical properties of stimuli. A study on numerical cognition by Davis (1984), for instance, reported number sensitivity in animals and showed that they could correctly select a set containing three objects from alternatives containing lower or higher number of stimuli, suggesting the use of an absolute responding rule (see also Taniuchi et al., 2016). In contrast, Brannon and Terrace $(1998 ; 2000)$ showed that animals trained to discriminate between different sets of numerosities can transfer previously learned ordinal information to new numerosities. A similar use of relative relationships has been also found in several spatial cognition studies investigating the transfer of previously learned landmark-to-goal distances to novel interlandmark distances (e.g., Kamil \& Jones, 2000; Spetch et al., 2003). These findings indicate that numerical and spatial information might be encoded in either absolute or relative terms or both of these two types of representations. The relation between these types of representations is not symmetric in the sense that a relative representation of quantities cannot exist without absolute representations. To know that one thing is twice as large as another or even that one thing is greater than the other requires that the absolute quantities first be encoded. Thus, the use of a 
relative representation implies that an absolute representation of quantity must be used to create a relative one.

In the present studies, we examined how these kinds of representations interact in the temporal domain. Although this question of how the passage of time is represented is not a novel one, previous literature has provided mixed evidence on whether and how relative and absolute temporal representations guide behavior, and studies typically argue for one type of temporal code over the other. Several theories of timing, including some of the neurobiologically-oriented ones, explicitly or implicitly assume that the brain encodes durations as disparate objects (e.g., Gershman \& Uchida, 2019; Hardy \& Buonomano, 2018; Karmarkar \& Buonomano, 2007; Matell et al., 2003; Petter et al., 2018). Attributing a categorical representation to a duration would imply that a 2-s duration is coded as one object category, whereas a 6-s duration is coded as another, different category. Such nominal representations would limit the brain's ability to perform basic operations on a set of durations and not allow a response associated with the fact that $6 \mathrm{~s}$ is longer than $2 \mathrm{~s}$ or shorter than $18 \mathrm{~s}$ (which would constitute a third object category). By contrast, other timing models (Church, 1984; Gibbon, 1977; Gibbon et al., 1984) assume that duration experiences are metrically encoded, suggesting that the brain can apply basic arithmetic operations and decide that an 18-s duration is not only greater than 6-s duration but that it is 3 times as long. From a measurement theory perspective (Stevens, 1946), if time is encoded on a ratio scale then other representations can be computed. In this view, multiple temporal representations might guide behavior including, categorical (nominal), relative (ordinal), and even metric (interval and ratio) representations of time.

There is much evidence absolute representations of time guide performance in duration discriminations. For example, Carvalho and Machado (2012) and Carvalho et al. (2016) initially 
trained pigeons in duration discrimination tasks with two time intervals and then altered one of the durations so the unchanged duration had a change in ordinal position. In the focal groups for example, an animal might have been trained to peck the green response key after a 6-s duration and peck the red response key after an 18-s duration. In a transfer test, the animal might still peck the green key after the 6-s cue but now has to peck the red key after a 2-s cue. In this way the mapping of the 6-s time to a particular response is preserved. However, what was previously the shorter cue (6 vs. 18s) has now become the longer one (6 vs. 2s). If the encoding of absolute time was guiding performance in the original discrimination then one would predict strong positive transfer to the second discrimination. Perhaps, nearly perfect transfer to the 6-s cue while the animal has to learn what to do to a 2-s cue. In the case of the 2-s cue, there might even be some negative transfer as the 2-s cue is closer to the 6-s than the 18-s cue of the original discrimination. This might result in an initial tendency to peck the 6-s key following both cues. If the animals had encoded the original discrimination with a relative code, then there ought to be negative transfer to the 6-s cue. This cue switched its relative identity from being the shorter cue in the first discrimination ( 6 vs. $18 \mathrm{~s}$ ) to being the longer cue in the second discrimination (6 vs. $2 \mathrm{~s})$. If the animal had learned to peck the green key to the shorter cue and the red key to the longer one, then once the animal discovered that $6 \mathrm{~s}$ was now the longer cue the performance ought to deteriorate as the animals starts to choose the red key based on the new relative duration of the 6-s cue. In the aforementioned studies from the Machado lab, subjects in general had high accuracy following the duration that was associated with the same response key in both training and transfer phases (i.e., preserved absolute mapping). In different experimental conditions where short and long durations were associated with same response keys in both phases (i.e., 
preserved relational mapping), subjects had low accuracy following the duration that was preserved from the training phase, for which they had to reverse the classification response.

However, a closer look at individual subjects' discrimination accuracy after transfer in the Carvalho and Machado (2012) study reveals that most subjects in one of the experimental conditions (Phase B, Upshift) did not benefit from the preserved absolute mapping. Furthermore, in both Carvalho and Machado (2012) and Carvalho et al. (2016) studies, although preserving the response location of a duration seemed to have helped with initial transfer in most cases, individual acquisition curves frequently followed a U-shaped pattern during the transition phase. That is, the discrimination accuracy for the cue duration with preserved response location started at a high level, but then dropped down significantly before the animals learned the new task rules. While the initial transfer is consistent with an absolute account of temporal control, the subsequent deterioration is not. Similarly, other studies employing temporal discrimination tasks have also yielded support for the hypothesis that at least some of what is encoded has to do with the absolute duration of events (e.g., Russell \& Kirkpatrick, 2007; Spinola et al., 2013), though perhaps not to the exclusion of a relative encoding.

There is also considerable behavioral evidence suggesting that organisms rely on relative temporal representations (e.g., Church \& Deluty, 1977; Fetterman et al., 1989, 1993; Wan et al., 2010). For example, in the Church and Deluty (1977) study, transfer to conditions that preserved the relative mappings were learned faster than those that did not. However, performance was aggregated across long and short stimuli, so specific ways in which absolute durations might have affected the transfer were not presented. In a clever test of the absolute and relative accounts, Zentall et al. (2004) trained two groups of pigeons on a temporal discrimination task where they first learned to discriminate a set of two time intervals ( 2 vs. 8 s or 4 vs. 16s). They 
were then tested on an additional temporal discrimination in which one of the durations was the geometric mean of the durations used in the first discrimination. Specifically, the shorter duration from the 4 vs. 16s discrimination represented the geometric mean of the previous, 2 vs. $8 \mathrm{~s}$ discrimination in one group. In the other group, the longer duration from the $2 \mathrm{vs} .8 \mathrm{~s}$ discrimination represented the geometric mean of the previous, 4 vs. 16 s discrimination. These intermediate durations were expected to produce indifferent choices based on the absolute encoding, as it is not obvious how an absolute account could predict positive or negative transfer to a value that is midway between the previous short and long durations. Moreover, from an associative strength point of view (e.g., Spence, 1938), the generalized excitatory tendencies to call the middle value long or short ought to be equal. Any generalized inhibition of the alternate response also ought to be equal. Thus, there is no basis for positive or negative transfer to the intermediate cue. Subjects ought to perform at chance levels when presented with intermediate durations. The results, however, indicated that the stimulus durations at the mean were grouped with the other duration based on their relative relationships (i.e., as being shorter or longer than the alternative) during training, demonstrating an example of relational learning (see also Molet \& Zentall, 2008 for similar findings in humans). When Maia and Machado (2009) tested pigeons in a duration bisection experiment similar to the Zentall et al. (2004) study, however, they found no evidence for the relative classification of durations.

Even though the exact reasons for these discrepancies in previous findings are not clear, there is a considerable amount of difference in training procedures, learning criteria, and analysis methods across studies. Aside from these methodological differences, however, the lack of consensus on the description of how time is encoded in different timing procedures might also indicate that animals form both absolute and relative temporal representations, not either one or 
the other. Previous research on how temporal information is utilized in associative learning indeed shows that animals are capable of both encoding the length of time intervals and comparing the durations of behaviorally relevant events to one another to guide behavior. It should be noted that even though the order in which the absolute and relative categorizations are formed is not known, one possibility is that animals first encode the absolute duration of events and then compare them while learning new temporal contingencies (e.g., Balsam \& Gallistel, 2009; Balsam et al., 2006).

To further probe the question of how time is encoded, we first trained mice to categorize the length of two time intervals as short or long in a temporal discrimination task and then examined how they transferred this discriminative behavior to novel cue durations and response locations in two different experiments (Table 1). Specifically, after training mice to correctly discriminate a duration pair, we either increased or decreased the length of one of the initial time intervals by a factor of 3 (Experiment 1 and Experiment 2, respectively) and/or switched the associated response locations (left or right lever). 


\section{Table 1}

Cue Durations and Corresponding Response Locations in All Training and Transfer Phases

\begin{tabular}{lccccc}
\hline & \multicolumn{2}{c}{ Experiment 1 (Upshift) } & & \multicolumn{2}{c}{ Experiment 2 (Downshift) } \\
\cline { 2 - 3 } \cline { 5 - 6 } Phases and conditions & Left Lever & Right Lever & & Left Lever & Right Lever \\
\hline Training (Phase 1) & $2 \mathrm{~s}$ & $6 \mathrm{~s}$ & & $6 \mathrm{~s}$ & $18 \mathrm{~s}$ \\
Transfer (Phase 2) & $2 \mathrm{~s}$ & $6 \mathrm{~s}$ & & $6 \mathrm{~s}$ & $18 \mathrm{~s}$ \\
$\quad$ Control & $6 \mathrm{~s}$ & $2 \mathrm{~s}$ & & $18 \mathrm{~s}$ & $6 \mathrm{~s}$ \\
Reversal & $6 \mathrm{~s}$ & $18 \mathrm{~s}$ & & $2 \mathrm{~s}$ & $6 \mathrm{~s}$ \\
Relative & $18 \mathrm{~s}$ & $6 \mathrm{~s}$ & & $6 \mathrm{~s}$ & $2 \mathrm{~s}$ \\
Absolute & & & & $4 \mathrm{~s}$ & $12 \mathrm{~s}$ \\
Transfer (Phase 3) & & &
\end{tabular}

Note. Opposite duration-lever assignment was used for half of the animals. Note that Experiment 1 consisted of only one transfer phase.

In Experiment 1 (Upshift), we started with a short duration pair and trained mice on 2 vs. $6 \mathrm{~s}$ discriminations. After they reached a high discrimination accuracy level, we divided the animals into four groups. In two of these groups (absolute and relative groups), we increased the short duration from $2 \mathrm{~s}$ to $6 \mathrm{~s}$ and the long duration from $6 \mathrm{~s}$ to $18 \mathrm{~s}$, thus preserving one of the cue durations (6 s) between both experimental phases. In the absolute group, the specific response location (left or right lever) associated with this unchanged, 6-s cue duration remained constant, such that if the mice were trained with the "2s/Left, 6s/Right" mapping, they were then tested with the "18s/Left, 6s/Right" mapping in the transfer phase. Because of the accompanying change in the alternate duration, the lever associated with the consistent mapping of absolute time (6 s) also experienced a reversal of relative mappings. That is, the preserved response location associated with the long cue of the training pair was now associated with the short cue of the transfer pair. Consequently, if the experienced durations are only coded in an absolute way (e.g., categorically), animals should be little affected by the shift in durations and response locations and still perform with high accuracy after transfer. However, if they rely on relative 
representations there should be negative transfer in this group, as the identity of the short and long levers during testing is reversed from what it was during training.

The relative group in Experiment 1 (Upshift), on the other hand, was tested with the same relative lever-duration mapping after transfer (e.g., short cue/left lever, long cue/right lever). As in the absolute group, the 6-s duration was preserved from the training to the transfer phase, such that after being initially trained with the " $2 \mathrm{~s} /$ Left; $6 \mathrm{~s} /$ Right" mapping, animals were tested with the "6s/Left, 18s/Right" mapping in the transfer phase. Since the identity of the correct short and long levers was preserved and the 6-s cue became the short cue of the transfer pair, animals had to change the classification response for the unchanged duration. Thus, if animals rely primarily on absolute representations, we should see negative transfer in the relative group. However, if the relative ordering of remembered durations is a property of temporal encoding, maintaining the relative mapping might facilitate the transfer to the new duration pair.

In the remaining two groups of Experiment 1, we did not change cue durations. In the reversal group, we only switched response locations in the transfer phase. If the old mapping was "2s/Left, 6s/Right" in Phase 1, then the new mappings were "6s/Left, 2s/Right" in Phase 2. Since, both the relative and absolute mappings had to be relearned in the reversal group, we thought this might make it the most difficult of the transfer conditions if both types of representations guide behavior. Finally, a control group was tested with same duration pairs and response locations in both training and transfer phases (e.g., 2s/Left, 6s/Right in both phases). We ran this last group of animals as a very conservative control to demonstrate that performance does not systematically change as a function of the amount of training.

In another experiment with a separate group of mice, we replicated the design of Experiment 1 (Upshift), except that in Experiment 2 (Downshift), we tested the animals in the 
reverse transfer order. Specifically, we initially trained all subjects with the "6s/Left, 18s/Right" mapping, and then divided animals into four groups and randomly assigned them to absolute, relative, reversal, and control groups as outlined above. In the relative and absolute groups, we preserved one of the cue durations (6 s) and changed the other cue duration by decreasing it by a factor of 3 (short cue: $2 \mathrm{~s}$, long cue: $6 \mathrm{~s}$ ), whereas the control and reversal groups continued to be tested with the 6 vs. $18 \mathrm{~s}$ duration pair. To address how relative and absolute information is utilized both during initial transfer and later as animals adjust to the transfer task, we analyzed animals' duration discriminations over the course of the transfer tests in both Upshift and Downshift experiments.

\section{Experiment 1}

\section{Method}

\section{Subjects}

Thirty-one naive male C57BL/6j mice (The Jackson Laboratory, Bar Harbor, ME, USA) were used in this experiment. Mice were approximately 9 weeks-old upon arrival. They were housed in groups of four or five and kept with 12:12h light-dark cycle. Experimental sessions were conducted during the light phase. The mice were maintained at $85-90 \%$ of their free-feeding weight. Water was available ad libitum in home cages. The experiments reported in this article were in accordance with the Columbia University and New York State Psychiatric Institute Institutional Animal Care and Use Committees and Animal Welfare regulations.

\section{Apparatus}


Sixteen operant chambers (ENV-307W; Med-Associates, St. Albans, VT) placed inside sound and light attenuating boxes were used in the experiment. A feeder trough was centered on one wall of the chamber and used to deliver liquid reward of evaporated milk $(0.01 \mathrm{ml})$. Two retractable levers (ENV-312-2W; $2.2 \mathrm{~cm}$ above the grid floor and $11 \mathrm{~cm}$ apart) were mounted on either side of the feeder trough. An audio speaker (ENV-324W) was used to deliver a tone (90 $\mathrm{dB}, 2500 \mathrm{~Hz})$ stimulus. A house light (ENV-315W) was located on the opposite wall, at the top of the chamber. A cooling fan was activated in each chamber at the start of an experimental session. The experimental protocol was controlled via Med-PC computer interface and Med-PC IV software located in an adjacent room. Behavioral events were recorded with a temporal resolution of $10 \mathrm{~ms}$.

\section{Procedure}

Magazine and lever press training. Lever press training consisted of two phases. On the first day of the first phase, animals were trained to consume the milk reward from the raised dipper which was lowered $9.5 \mathrm{~s}$ after the first head entry. A variable inter-trial interval (ITI) selected from a list of durations with a mean of $30 \mathrm{~s}$ was presented after reward delivery. The session ended either after 30 min or 20 dipper presentations, whichever came first. On the subsequent day, animals were trained with the same protocol except this time the dipper was presented for $8 \mathrm{~s}$. All mice were able to collect 20 rewards in both sessions. In the second phase, animals were trained to press a lever to earn the reward. A lever press during the first $6 \mathrm{~s}$ of lever insertion resulted in immediate reward for $5 \mathrm{~s}$ (Fixed-ratio 1 [FR1]). The milk reward was also presented when the lever has been extended for $6 \mathrm{~s}$ without a press (Fixed-time $6 \mathrm{~s}$ [FT6s]). In both cases, the lever was retracted the moment the dipper was raised, and then a random ITI selected from an exponential distribution with a mean of $45 \mathrm{~s}$ was presented after $5 \mathrm{~s}$ of food 
delivery. Sessions ended when mice earned 60 reinforcements or after 60 min elapsed, whichever occurred first. Mice continued receiving sessions like this for a maximum of five sessions until they earned 30 rewards in two consecutive sessions. If they could not attain the performance criterion, they were then switched to FR1 trials in which there was no reward delivery independent of lever pressing. That is, the animals were required to press a lever to earn the reward. Otherwise, the levers stayed extended until the session ended after 60 minutes. The ITI and performance criterion were the same as in FR1\&FT6s trials. On average, lever press training lasted 8.32 sessions $(S D=1.80$, range $=7-14)$. Sessions occurred once a day, seven days per week both during this and all other experimental phases.

Duration Discrimination Training, Phase 1. Following lever press training, mice were trained on a duration discrimination task in which they learned to correctly report an experienced duration as short or long to earn milk rewards. Specifically, mice were required to press either the left or the right lever after the presentation of an auditory tone for one of the two randomly selected durations (short: $2 \mathrm{~s}$, long: $6 \mathrm{~s})$. Half of the mice $(n=16)$ were trained to press the right lever after the long tone and the left lever after the short tone presentation, whereas the remaining mice $(n=15)$ were trained with the reverse lever and duration mapping. Duration discrimination training consisted of six phases with increasing complexity from the presentation of only a single duration and the corresponding lever to a standard discrimination procedure to facilitate the acquisition of temporally controlled behavior. We mainly followed the training protocol described in Ward et al. (2009; Experiment 2), with slight modifications. On Day 1 of the single-lever, single-duration program, after the presentation of the short duration ( $2 \mathrm{~s}$ ) only the lever associated with a correct short categorization was extended. A press on this lever within $10 \mathrm{~s}$ led to the retraction of the lever and was rewarded with 5-s of reinforcement delivery. Late 
responses resulted in trial termination. On Day 2, only the long duration (6 s) and the corresponding lever were presented. Duration-lever mapping was counterbalanced across subjects. On Days 3-6, a single lever, two-duration program was in effect, where only one lever was extended after the presentation of one of the two, randomly selected durations ( 2 or $6 \mathrm{~s})$. If the cue duration corresponded to the presented lever, presses were reinforced. If the cue duration did not correspond to the presented lever, responses did not lead to reward delivery. Lever identity (left or right) was constant within a session and was counterbalanced across days.

On Days 7-8, the mice completed the interspersed single-lever, single-duration program in which each of the two durations were randomly presented with their respective corresponding levers. The mice then completed 3 sessions of $50 \%$ choice response trials $(50 \%$ single-lever and single-duration trials), 2 sessions of $75 \%$ choice response trials (25\% single-lever and singleduration trials), and 5 sessions of $100 \%$ choice response trials programs which had an increasing presentation probability of trials that involved the extension of both levers after tone presentation and required animals to choose the lever that was associated with a cue duration to earn a reward. On choice trials, short and long durations were randomly presented, and both levers were retracted upon a lever press. Moreover, an incorrect categorization was followed by a correction trial in which the house light was illuminated for $1 \mathrm{~min}$, and following an ITI (with a mean of 45 s) another choice response trial with the same cue duration initiated. The house light was illuminated only during correction trials. The correction procedure was then removed, and five more sessions of $100 \%$ choice response trials were completed. Sessions ended when mice earned 40 reinforcements or after 70 min elapsed, whichever occurred first. Mice continued the duration discrimination training until their discrimination accuracy, quantified by the proportion of correct responses, for each reference duration was at least $75 \%$ for two consecutive sessions. On 
average, the mice required 2.94 more sessions to reach criterion $(S D=1.90$, range $=2-11)$, and the whole duration discrimination training lasted between 24 and 34 sessions.

\section{Duration Discrimination Upshift Transfer Test, Phase 2. After successfully}

completing the duration discrimination training, mice were randomly divided into four groups and transferred to conditions in which target intervals and corresponding response locations were systematically manipulated. In Phase 2, we switched the response locations and/or durations of cues in three of the four groups (Table 1). In the first group (control, $n=8$ ), neither the durations nor the response locations were altered. The second group (reversal, $n=7$ ) was tested with the original target intervals ( 2 vs. 6 s), but the lever-duration mapping was reversed. Consequently, if animals were originally trained to press the left lever following the short, 2-s tone and the right lever following the long, 6-s tone, they now had to press the right lever after the short cue and the left lever after the long cue presentation. For this group, both the absolute and relative mappings are disrupted. In the remaining two conditions, the short cue duration was increased from $2 \mathrm{~s}$ to $6 \mathrm{~s}$ (former long duration) and long cue duration was increased from $6 \mathrm{~s}$ to $18 \mathrm{~s}$. These shifts in durations ensured that the ratio between short (S) and long (L) cues was kept constant at 1:3 in both training and transfer phases. The relative group $(n=8)$ was required to utilize the original lever assignment for the relative duration judgment, such that they had to press the same short-lever after the short (now 6 s) cue presentation and press the same longlever following the 18 -s duration. In the absolute group $(n=8)$, on the other hand, mice had to press the same lever following the 6-s cue in both experimental phases. This meant that the lever that was formerly associated with the longer duration was the correct response for the new short duration. During the transfer phase, all mice completed 10 sessions and then continued until they 
achieved at least $75 \%$ discrimination accuracy in two consecutive sessions (resulting in the completion of a minimum of 12 sessions by all animals).

\section{Data analysis}

Changes in temporal categorizations in response to the manipulations of cue durations and response locations were analyzed with respect to duration discrimination accuracy, quantified by the proportion of correct responses, and days to criterion. When necessary, HolmBonferroni corrections were used to adjust for all multiple comparisons. An alpha level of .05 (two-tailed) was used for all statistical tests. Data analyses were run in MATLAB (Mathworks, Natick, MA) and JASP (Version 0.12.2).

\section{Transparency and Openness}

The experiments reported in this article were not formally preregistered. Requests for the data or materials can be sent via email to the corresponding author.

\section{Results}

There was no significant difference in discrimination accuracy between groups during the initial training prior to transfer, One-way ANOVA: $F(3,27)=0.42, p=.74$. To investigate the effects of altering cue durations and response locations on duration discriminations, we analyzed the discrimination performance across the first 12 post-transfer sessions (Figure 1). Our results indicated that the proportion of correct responses in all groups, except the controls, decreased dramatically in the first session after transfer. Reversal of the cue duration-lever assignment had a particularly detrimental effect on average accuracy. Recovery of discrimination accuracy in the relative groups occurred very rapidly and was more complete than in the absolute and reversal groups even after 12 days of training. To examine these changes in discrimination accuracy and learning speeds more in detail, we first compared the proportion of correct responses in the first 
session of the transfer phase and found evidence for positive transfer based on both absolute and relative representations of time. As can be visualized in Figure 1 insets, the overall average proportion of correct trials in the absolute and relative groups dropped from approximately .90 pre-transfer to .50 in the first post-transfer session. Discrimination performance of the reversal group deteriorated even further, and the average accuracy dropped approximately to .20. As expected, the control groups' classification accuracy did not change. One-way ANOVAs supported these findings and revealed an overall difference in the proportion of correct choices across groups, $F(3,27)=90.30, p<.001, M S E=0.58, n^{2}=0.91$. Holm-Bonferroni corrected post hoc comparisons showed that all groups differed from one another except for the relative and absolute groups $(p=.49$, all other $p \mathrm{~s}<.001)$. 


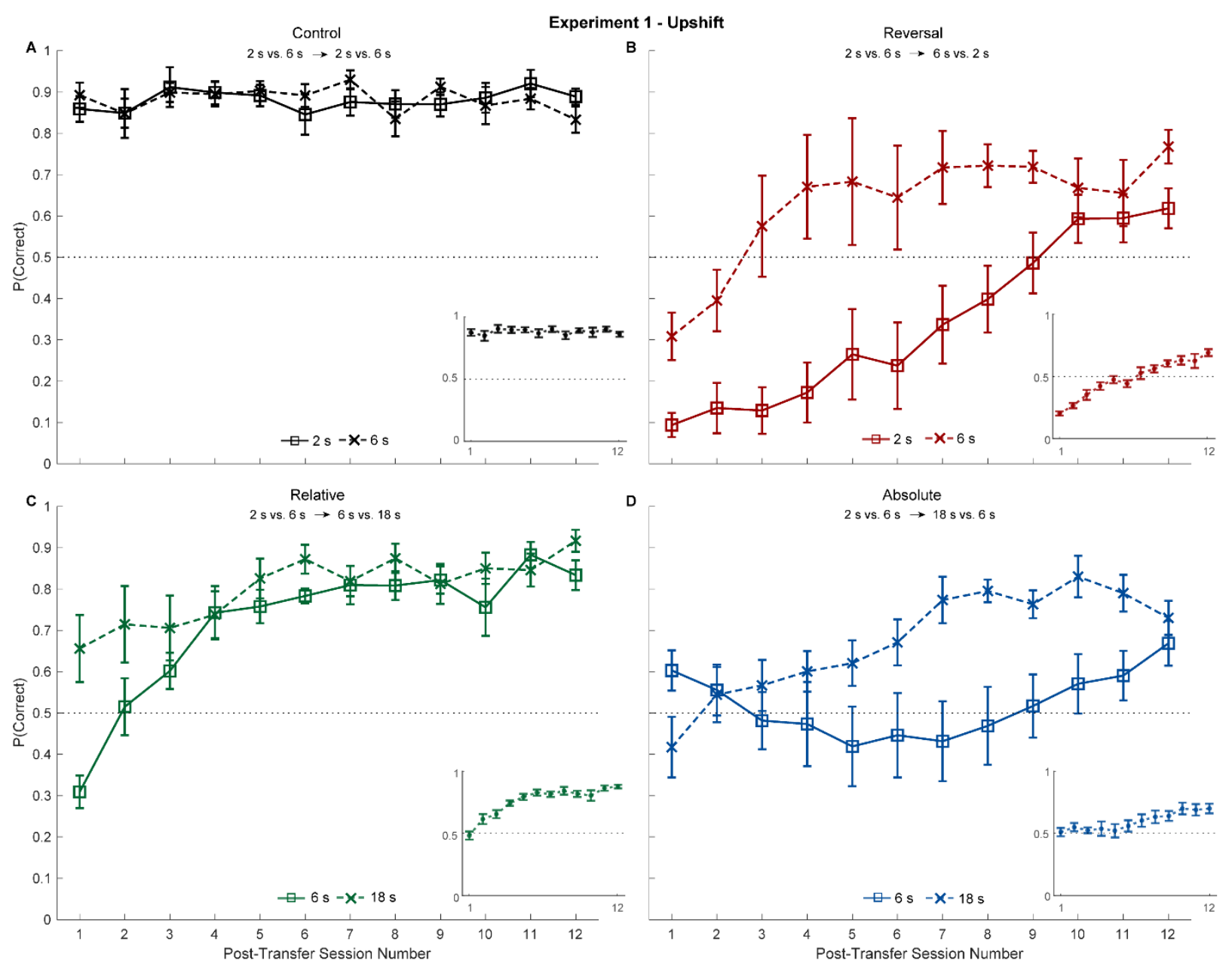

Figure 1. Proportion of correct responses on short and long trials after transfer shown separately for each experimental group in Phase 2 of Experiment 1. The insets show the average proportion of correct responses for all trials. Error bars show SEM. Note that we only included the first 12 post-transfer sessions in this graph, as some animals completed Phase 2 in 12 sessions, whereas others required more prolonged training.

The overall group averages conceal systematic differences in the proportion of correct choices on short and long trials (Figure 1). Also, averaging across subjects within groups 
conceals theoretically interesting between-subject differences in responses to the same change. To better address these issues, we first broke down the averages by cue durations in the posttransfer sessions (Figure 1). Reversal of the cue duration-correct lever mapping drove average accuracy below chance following both 2- and 6-s cues (Figure 1B). In the relative group (Figure 1C), by contrast, the first post-transfer session performance following the novel, 18-s cue duration was on average significantly above chance. On the other hand, when subjects in the relative group were presented with the old, already familiar cue duration $(6 \mathrm{~s})$ with a new mapping to the correct lever, the initial performance was on average below chance. We observed the reverse pattern in the absolute group and found that the proportion of correct trials was above chance following the presentation of the 6-s cue, while performance dropped slightly below chance following the 18-s cue duration (Figure 1D).

To evaluate the consistency of these observations about the first transfer session, we examined the proportion of correct responses only for the 6-s cue which was the common duration across all four groups, and found significant differences (One-way ANOVA; $F(3,27)=$ 39.93, $p<.001, M S E=0.61, n^{2}=0.82$ ). Pairwise comparisons revealed that all groups performed worse than the control group $\left(M_{\text {control }}=0.89, S D_{\text {control }}=0.09\right.$; all $\left.p \mathrm{~s}<.001\right)$. Importantly, even though the absolute group's average proportion correct was only at $.60(S D=$ 0.14), they still performed better than the relative and reversal groups whose accuracy levels were well below chance (both $p \mathrm{~s}<.001)$ and did not differ from one another $\left(M_{\text {relative }}=0.31\right.$, $\left.S D_{\text {relative }}=0.11 ; M_{\text {reversal }}=0.31, S D_{\text {reversal }}=0.15 ; p=0.99\right)$. These differences in discrimination accuracy indicate that the absolute group benefited from the preserved absolute mapping of the 6-s cue during initial transfer. 
We then analyzed the absolute and relative groups' discrimination accuracy following the novel, 18-s cue duration in the first post-transfer session (Figure 1). The findings revealed that the relative group performed well above chance, whereas the proportion of correct responses in the absolute condition was below chance following the 18 -s cue $\left(M_{\text {relative }}=0.66, S D_{\text {relative }}=0.23\right.$; $M_{\text {absolute }}=0.42, S D_{\text {absolute }}=0.21$; independent samples $t$-tests; $t(14)=-2.18, p=.047, d=-1.09$ ). These findings suggest that even though both groups of mice were tested with a novel duration in Phase 2, maintaining the relative duration-lever mapping before and after the shift resulted in an immediate and appropriate shift in performance following the novel, 18-s cue duration.

As can be inferred from Figure 1, altering the mapping of relative and absolute durations to response locations also affected how fast the new task was learned in Phase 2. The relative group's discrimination accuracy increased and reached asymptote only in a few sessions after transfer, whereas the reversal and absolute groups required many more sessions to re-establish baseline accuracy. Since we tested animals (including those in the control group) for a minimum of 10 days before starting to assess their discrimination accuracy, we included those 10 days in our first analysis of learning speeds. As expected, our one-way ANOVA on the number of sessions the animals took to achieve at least $75 \%$ overall discrimination accuracy for two consecutive sessions after completing at least 10 sessions indicated that relearning speeds indeed differed across groups, $F(3,27)=7.10, p=.001, M S E=86.21, n^{2}=0.44$. Specifically, the absolute and reversal groups $\left(M_{\text {absolute }}=18.38, S D_{\text {absolute }}=5.83 ; M_{\text {reversal }}=19.00, S D_{\text {reversal }}=2.94\right.$; $p=1.00)$ were slower than the relative and control groups $\left(M_{\text {relative }}=13.38, S D_{\text {relative }}=2.13\right.$; $\left.M_{\text {control }}=12.50, S D_{\text {control }}=0.93 ; p=1.00\right)$ to achieve high duration discrimination accuracy (all other $p s \leq 0.02$ ). 
Although we tested animals for at least 10 sessions before assessing their performance, visual inspection of individual acquisition curves (see Figures S1 and S2 in the supplementary text) indicates that some animals did not require such lengthy training. Furthermore, we also found that the change in discrimination accuracy for the 6-s cue that had the preserved response location pre- and post-transfer followed a U-shaped pattern for most subjects in the absolute condition (see also Figure 1D), whereas the accuracy levels for both short and long duration discriminations increased monotonically in other experimental groups. Consequently, in the next set of analysis we aimed to better characterize the differences in relearning speeds both within and across groups. To this end, we calculated the number of training days that each animal needed to achieve at least $75 \%$ accuracy in their categorizations of short and long durations separately for two consecutive sessions (Figure 2). 


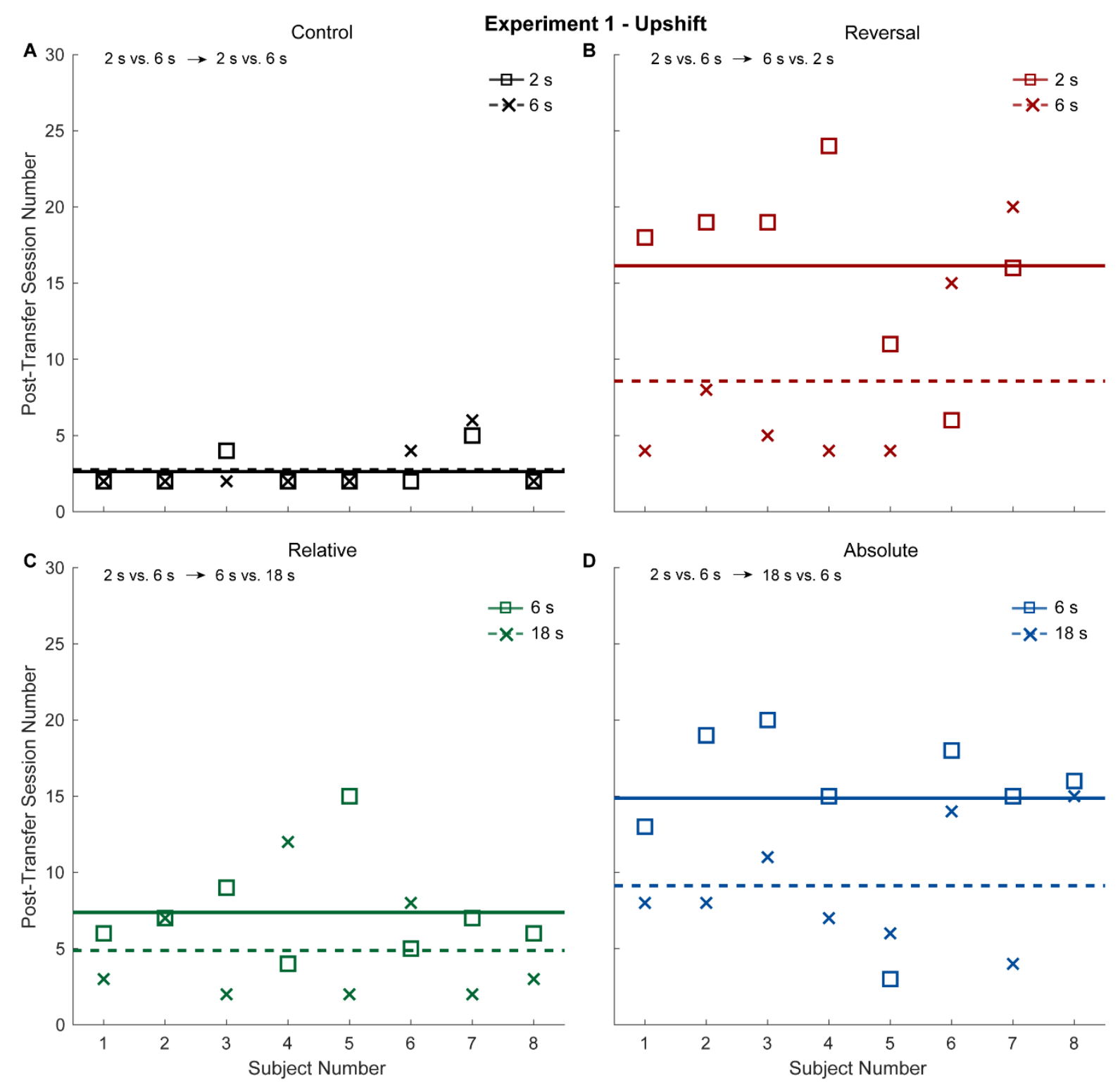

Figure 2. Number of sessions required to perform at $\geq 75 \%$ accuracy for two consecutive sessions shown separately for short and long cue durations in Phase 2 transfer test. Lines (solid: short duration, dotted: long duration) show the mean session numbers and symbols (square: short duration, cross: long duration) illustrate individual subjects' data. 
Our mixed ANOVA number of training days that each animal needed to achieve at least $75 \%$ accuracy revealed significant main effects of Duration, $F(1,27)=11.43, p=.002, M S E=$ $237.88, n_{p}^{2}=0.30$, and Group, $F(3,27)=22.88, p<.001, M S E=342.66, n_{p}^{2}=0.72$, as well as a non-significant interaction, $F(3,27)=2.14, p>.05, M S E=44.46, n_{p}^{2}=0.19$. Pairwise comparisons conducted for the main effects of Group and Duration revealed that the reversal and absolute groups required similar amount of training $(p=.80)$ and were slower to achieve $75 \%$ correct choices than the other two groups (all $p \mathrm{~s}<.001$ ). The difference between the relative and control groups was small, though statistically significant $(p=.04)$. Further, long durations were learned faster than short durations in the Upshift experiment $(p=.004)$. Although the groups followed these general trends, it is worth noting that Sub \#5 in the absolute group had a very quick recovery of correct responding to both short and long cue durations, which was highly comparable to the performance of the relative groups.

\section{Discussion}

Our findings indicate that both absolute and relative representations can be used by mice in this temporal discrimination task. When the mapping between relative duration and response was preserved, even when there was an initial decrement in performance, subjects rapidly recovered high levels of accuracy. When the mapping between absolute duration and response was maintained, there was an initial positive transfer to new conditions. However, over the course of next several sessions, performance to the preserved cue duration deteriorated before the animals in the absolute group re-established baseline discrimination accuracy. The deterioration of performance suggests that when both representations are available and put in conflict, preserving the relative identity of the short and long levers has a greater long-term impact on the animals' ability to adapt to new conditions than preserving the mapping between an absolute 
duration and the associated response. Finally, preventing them from relying on either type of temporal representation by reversing the duration and lever assignments, as in the case of the reversal group, resulted in below chance accuracy for both short and long durations as well as a slow recovery in performance. Taken together, these findings illustrate that animals encode event durations in both absolute (e.g., $2 \mathrm{~s}$ vs. $6 \mathrm{~s}$ ) and relative terms (shorter or longer) in a temporal discrimination task.

\section{Experiment 2}

To further investigate the nature of temporal representations, we tested a new set of 32 adult mice in the duration discrimination tasks with the reverse order of Experiment 1 (Upshift) and different sets of durations across three experimental phases. Specifically, we initially trained all subjects with the "6s/Left, 18s/Right" mapping, and then divided them into four groups in Phase 2. In the relative and absolute groups, we preserved one of the cue durations $(6 \mathrm{~s})$ and changed the other cue duration by decreasing it by a factor of 3, whereas the control and reversal groups continued to be tested with the 6 vs. 18 s duration pair. To further investigate absolute and relative temporal representations, we then tested all animals in another experimental phase with two novel durations (4 vs. $12 \mathrm{~s}$ ) that had the same relative duration-lever mapping as in Phase 2. These new durations in Phase 3 were selected such that one of them was the arithmetic mean of a previously learned duration pair in Phase 2. Based on absolute temporal control, we expected that the novel, intermediate duration would create ambiguity and lead to a deterioration in discrimination accuracy (Molet \& Zentall, 2008; Zentall et al., 2004) following the 12-s cue in the control and reversal groups of Phase 2, and following the 4-s cue duration in the relative and absolute groups of Phase 2. Based on relative temporal control, however, these changes in absolute time intervals were expected not to lead to any significant changes in discrimination 
accuracy and the speed of acquisition, as the short and long durations were associated with the same levers.

\section{Method}

\section{Subjects and apparatus}

Thirty-two naive male C57BL/6j mice (The Jackson Laboratory, Bar Harbor, ME, USA) were used in this experiment. The equipment used in this experiment was identical to that in Experiment 1.

\section{Procedure}

Lever Press Training. Procedural details were the same as in Experiment 1. On average, lever press training lasted 9.22 sessions $(S D=1.83$, range $=4-14)$.

Duration Discrimination Training, Phase 1. The training protocol was the same as in Experiment 1 except that the target intervals were 6 and $18 \mathrm{~s}$ in Experiment 2. Mice completed the last phase of duration discrimination training in an average of 16.13 sessions $(S D=5.27$, range $=12-29)$.

Duration Discrimination Downshift Transfer Test, Phase 2. As in Experiment 1, animals were equally divided into four groups, each tested with a different temporal and/or spatial contingency. Transfer in Experiment 2 was tested in the opposite direction of Experiment 1, such that instead of upshifting the absolute and relative groups' cue durations, in Experiment 2 the cue durations went from 6 and $18 \mathrm{~s}$ during initial training to 2 and $6 \mathrm{~s}$ during the transfer test. Control and reversal mice were tested with 6 and $18 \mathrm{~s}$ in both phases. Please refer to Table 1 for a complete description of transfer conditions. Performance criterion was the same as in Experiment 1 in this and subsequent phases. One mouse in the absolute group did not meet the performance criterion, and for that mouse transfer training was terminated after 35 sessions. 
Duration Discrimination Transfer Test, Phase 3. Following Phase 2 an additional transfer test was conducted. To examine transfer to a new set of absolute times while preserving the mapping of relative times to responses, all subjects were tested with a new set of durations: 4 and $12 \mathrm{~s}$. These values were the arithmetic means of the duration pairs used in Phase 2. Specifically, the 12-s cue was the arithmetic mean of the 6- and 18-s cue durations learned by the control and reversal mice (hereafter referred to as Group MeanLong, $_{n}=16$ ), and the 4-s cue was the arithmetic mean of the 2- and 6-s cues that were used for the relative and absolute mice (referred to as Group MeanShort $_{\text {, }}=16$ ). The relative mapping was the same as in the previous phase. Since the discrimination performance stabilized earlier than 12 sessions in the relative group of Experiment 1 (Figure 1C), the mice completed a minimum of only seven sessions instead of 12 sessions.

\section{Results}

There was no significant difference in discrimination accuracy between groups during the initial training prior to transfer, One-way ANOVA, $F(3,28)=1.81, p=.17$. Our analysis of the average discrimination performance across the first 12 post-transfer sessions in Experiment 2 also revealed a deterioration in duration discrimination performance following the shifts in cue durations and/or response locations (Figure 3 insets). We again found that the reversal group's average discrimination accuracy was below chance level, whereas the absolute and relative groups' overall discrimination accuracy dropped down only to chance level, as initial

performance in the latter two groups was a mixture of positive transfer to one cue and negative transfer to the other. One-way ANOVAs supported these findings and revealed an overall difference in the proportion of correct choices across groups in the first post-transfer session, $F(3,28)=214.30, p<.001, M S E=0.50, n^{2}=0.96$. Follow-up comparisons showed that all 
groups differed from one another except for the relative and absolute groups $(p=.15$, all other $p \mathrm{~s}$ $<.001)$.
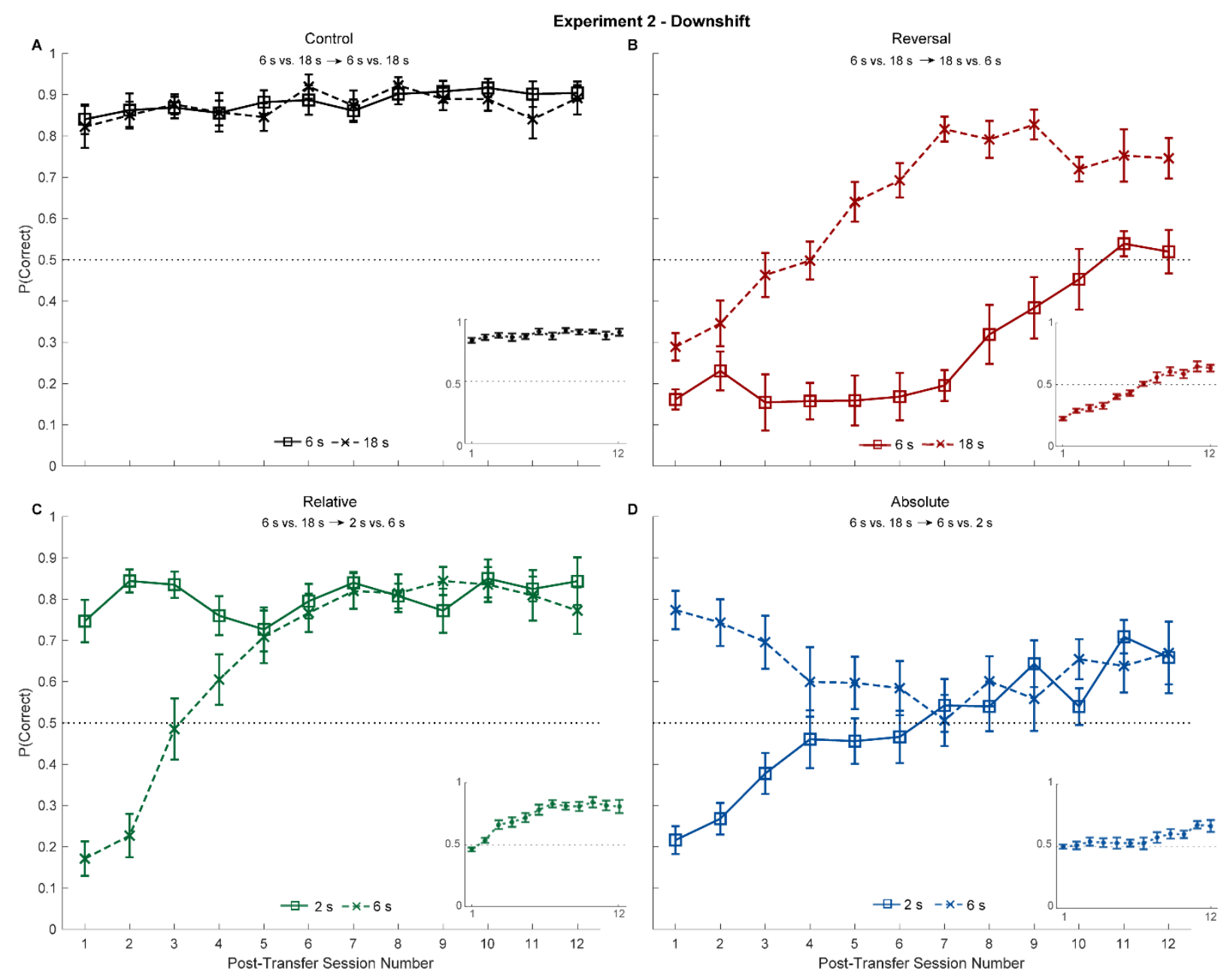

Figure 3. Proportion of correct responses on short and long trials after transfer shown separately for each experimental group in Phase 2 of Experiment 2. The insets show the average proportion of correct responses for all trials. Error bars show SEM. Note that we only included the first 12 post-transfer sessions in this graph, as some animals completed Phase 2 in 12 sessions, whereas others required more prolonged training. 
As in Experiment 1, when we broke down the averages by cue durations (Figure 3), we found that reversing the cue duration-correct lever mapping drove average accuracy below chance following both the 6- and 18-s cues in the first post-transfer session. In the relative group, by contrast, the discrimination performance following the novel 2-s cue duration was on average significantly above chance, whereas when subjects were presented with the old, 6-s cue duration with a new response mapping, their discrimination performance was below chance. Accuracy on both durations was high after a few sessions for subjects in the relative group. We observed the reverse pattern in the absolute group and found that the proportion of correct trials was above chance following the old, familiar 6-s duration, while performance dropped below chance following the novel 2-s cue duration. As training progressed performance to the 6-s duration deteriorated despite the initial positive transfer. In the absolute group it again took many sessions for performance to approach baseline accuracy in both cues.

To evaluate these observations, we examined the proportion of correct responses during the first post-transfer session for the 6-s cue which was the common duration across all four groups. Our one-way ANOVA results indicated a significant overall difference in accuracy on 6s trials, $F=(3,28)=95.31, p<.001, M S E=1.10, n^{2}=0.91$ Importantly, the absolute group was able to benefit from preserving the response location associated with the 6-s cue, as the subjects' average discrimination accuracy level was at $.77\left(S D_{\text {absolute }}=0.13\right)$, and not significantly different than that of the control group $\left(p=.45 ; M_{\text {control }}=0.84, S D_{\text {control }}=0.10\right)$ in the first post-transfer session. Pairwise comparisons indicated that these two groups performed significantly better on 6-s trials than both the relative and reversal groups (all ps $<.001$ ) whose accuracy levels were well below chance and did not differ from one another $\left(p=.86 ; M_{\text {relative }}=\right.$ $\left.0.17, S D_{\text {relative }}=0.12 ; M_{\text {reversal }}=0.16, S D_{\text {reversal }}=0.07\right)$. 
We then analyzed the absolute and relative groups' discrimination accuracy following the novel, 2-s cue in the first post-transfer session (Figure 3). We found that the relative group had highly accurate discriminations $(M=.75, S D=0.15)$ to the novel cue, whereas the absolute group's accuracy was well below chance following the 2 -s cue $\left(M_{\text {absolute }}=.22, S D_{\text {absolute }}=0.10\right.$; independent samples $t$-test; $t(14)=-8.64, p<.001, d=-4.32)$. These findings again show that preserving the relative identity of the short and long levers before and after the shift resulted in immediate and appropriate performance following the novel cue duration.

We then examined how fast the new task was learned in Phase 2 (also see Figures S3 and S4 in the supplementary text for individual acquisition curves). Similar to Experiment 1, the relative group's discrimination accuracy increased and reached asymptote in only a few sessions after transfer, whereas the reversal and absolute groups required more extensive training to approach baseline accuracy. As expected, our one-way ANOVA analysis on the number of sessions the animals took to achieve at least $75 \%$ overall discrimination accuracy for two consecutive sessions after completing at least 10 sessions indicated that relearning speeds differed across groups, $F(3,28)=12.34, p<.001, M S E=203.95, n^{2}=0.57$. Specifically, the absolute and reversal groups $\left(M_{\text {absolute }}=22.75, S D_{\text {absolute }}=6.56 ; M_{\text {reversal }}=21.63, S D_{\text {reversal }}=\right.$ $3.29 ; p=.58)$ were slower than the relative and control groups $\left(M_{\text {relative }}=14.75, S D_{\text {relative }}=3.41\right.$; $\left.M_{\text {control }}=12.50, S D_{\text {control }}=0.76 ; p=0.56\right)$ to achieve high duration discrimination accuracy (all other $p \mathrm{~s}<0.01)$.

When we calculated the number of training days that the animals needed to achieve at least $75 \%$ accuracy in their short and long duration categorizations separately for two consecutive sessions (Figure 4), we again found that in the relative group, the positive transfer for the novel, 2-s cue was almost immediate. The 6-s cue took several days to reach high 
accuracy levels. In the absolute group, there was enough initial positive transfer to the 6-s cue that the criterion was met almost immediately for that duration. In contrast, it took extensive training of the 2-s cue to reach criterion in that group. In the reversal group, both durations had to be retrained extensively to achieve high accuracy, though it took longer to train the 6-s cue than the 18-s one. Further, as in the Upshift experiment, most animals in the reversal and absolute groups learned to correctly categorize long durations faster than short durations. Our mixed ANOVA on the number of sessions needed to reach the $\geq 75 \%$ accuracy provided support for these conclusions and revealed significant main effects of Duration, $F(1,28)=18.57, p<.001$, $M S E=315.06, n_{p}^{2}=0.40$, and Group, $F(3,28)=25.51, p<.001, M S E=517.60, n_{p}^{2}=0.73$, as well as a significant interaction, $F(3,28)=17.48, p<.001, M S E=296.60, n_{p}^{2}=0.65$. Follow-up simple main effects analyses showed that the speed with which the short versus long durations was learned varied within each group (all $p$ s $<.02)$ except the control group $(p=.44)$, and that there was an overall difference in how fast the cue durations were learned across groups (short cue: $p<.001$; long cue: $p=.045)$. 


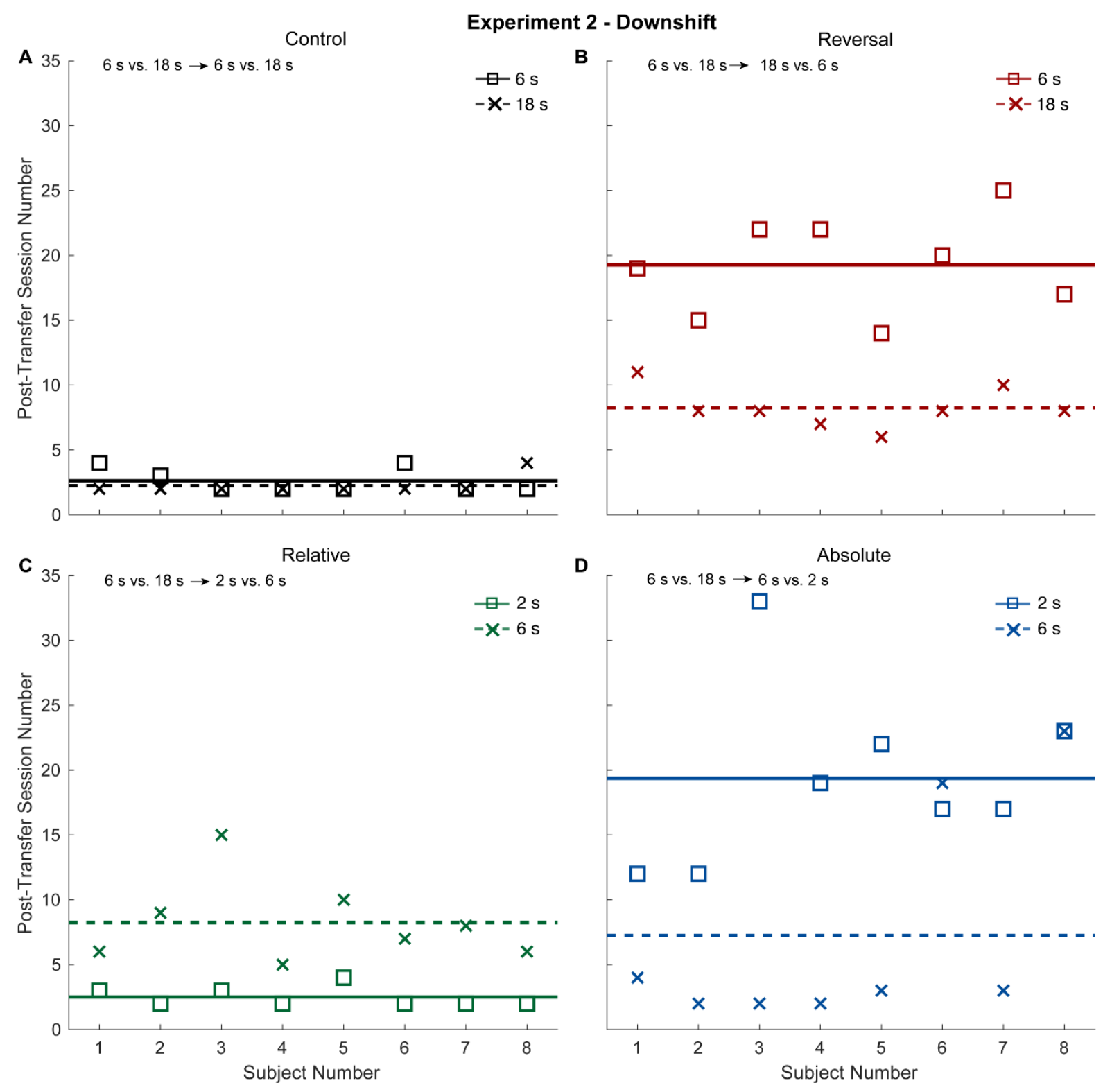

Figure 4. Number of sessions required to perform at $\geq 75 \%$ accuracy shown separately for short and long cue durations in Phase 2 transfer test. Lines (solid: short duration, dotted: long duration) show the mean session numbers and symbols (square: short duration, cross: long duration) illustrate individual subjects' data. 
Finally, after completing the transfer test with either the 6 vs. 18s (control and reversal) or the 2 vs. 6 s (relative and reversal) duration pairs, we tested the animals in one final transfer condition with a new set of durations (4 vs. 12 s) in Phase 3. In all groups, one of these time intervals was the arithmetic mean of a previously learned duration pair in Phase 2. If absolute times control responding, we expected one element of this new duration pair to create ambiguity and drive the discrimination accuracy down to chance level (Molet \& Zentall, 2008; Zentall et al., 2004). This should occur for the 12-s cue duration in Group MeanLong $_{\text {that }}$ was previously tested with 6 and $18 \mathrm{~s}$ (control and reversal groups of Phase 2), and for the 4-s cue duration in Group $_{\text {MeanShort }}$ that was previously tested with 2 and $6 \mathrm{~s}$ in Phase 2 (relative and absolute groups of Phase 2). Despite these changes in absolute time intervals, however, we hypothesized that preserving the relative mapping would result in rapid acquisition. The 6-s short time was shifted

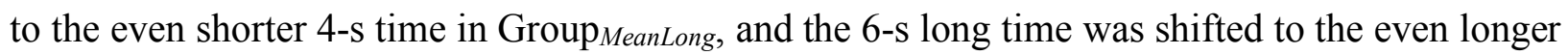

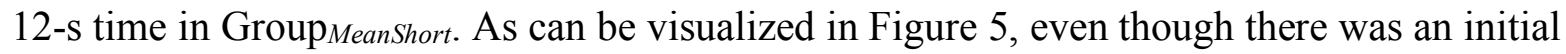
drop in discrimination accuracy for the ambiguous cue durations, the performance was still above chance level, and further improved and stabilized after only one or two days of training with the new duration pair. 

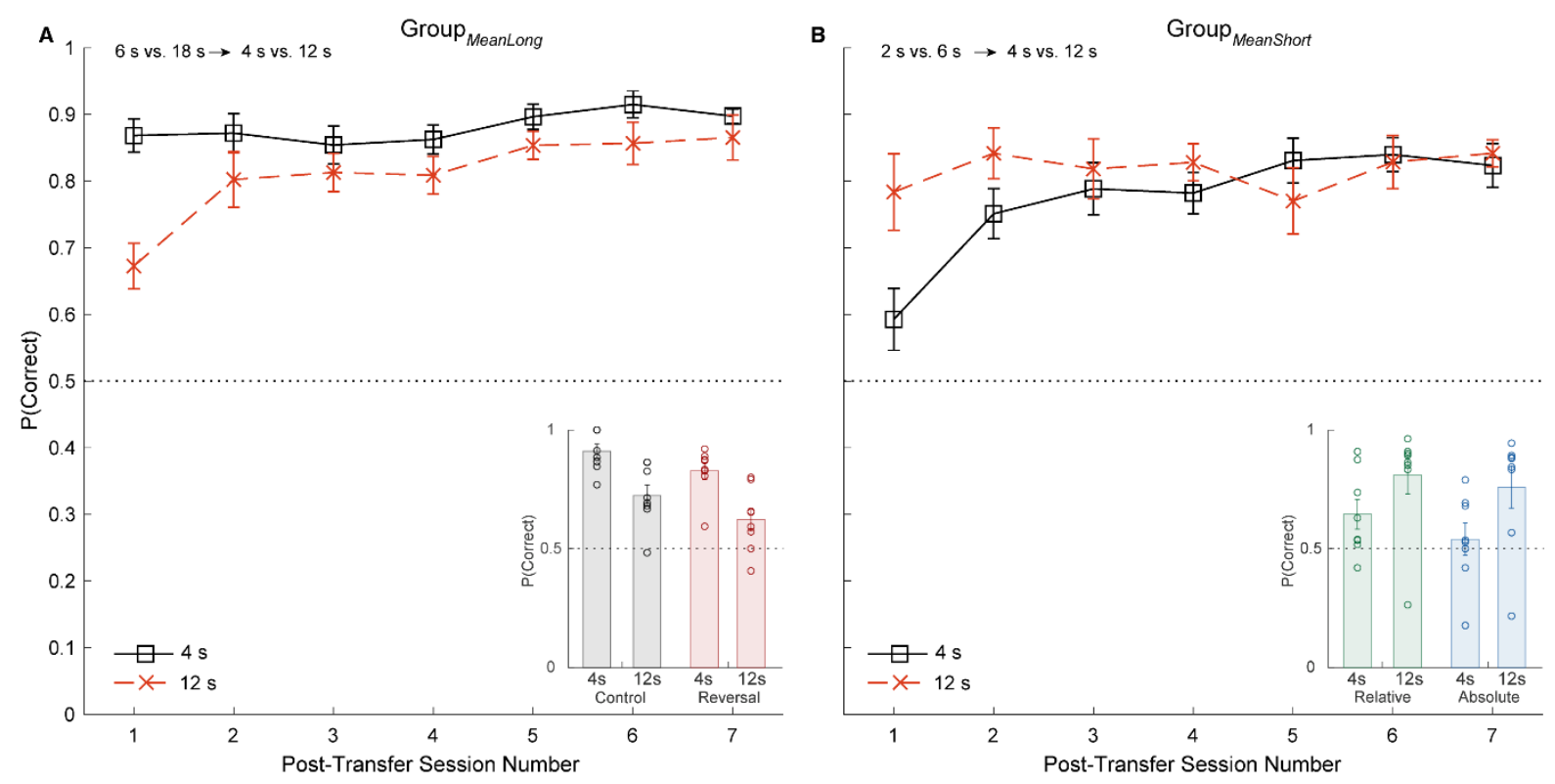

Figure 5. Proportion of correct responses on short and long trials after transfer shown separately

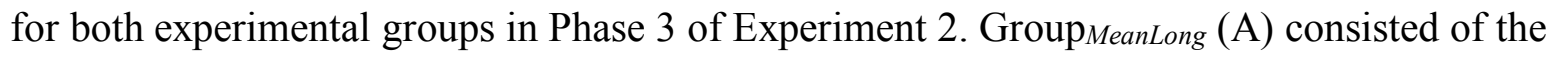
control and reversal groups of Phase 2, Group MeanShort $_{\text {(B) }}$ consisted of the relative and absolute groups of Phase 2 (refer to the inset plots for each sub-groups' discrimination accuracy in the first post-shift session). Note that we only included the first 7 post-transfer sessions in this graph, as some animals completed Phase 3 in 7 sessions, whereas others required more prolonged training. Error bars show SEM.

A mixed ANOVA analyzing the proportion of correct responses on short ( $4 \mathrm{~s})$ and long (12 s) trials in the first transfer session of Phase 3 revealed a significant main effect of Group, $F(1,30)=4.53, p=.04, M S E=0.11, n_{p}^{2}=0.13$, and a Duration x Group interaction, $F(1,30)=$ $17.55, p<.001, M S E=0.60, n_{p}^{2}=0.37$. Simple main effects analyses showed that the proportion of correct responses for the short cue (4s; $p<.001)$, but not for the long cue $(12 \mathrm{~s} ; p=.11)$, 
differed between two groups. We also found significant differences in the accuracy of short and long cue categorizations both in $\operatorname{Group}_{\text {MeanLong }}(p<.001)$ and $\operatorname{Group}_{\text {MeanShort }}(p=.04)$.

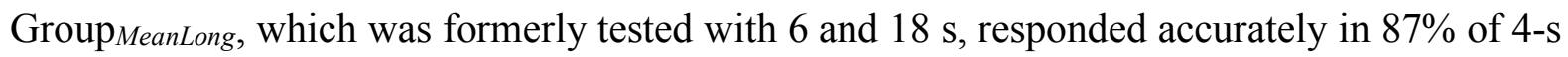
trials $(S D=.10)$, whereas the group-average accuracy dropped to $67 \%$ on the now ambiguous 12-s trials $(S D=.14)$. On the other hand, Group ${ }_{\text {MeanShort, }}$ which was tested with 2 and $6 \mathrm{~s}$ in Phase 2, showed the opposite pattern during the final transfer test and performed accurately in $78 \%$ of 12 -s trials $(S D=.23)$, whereas the average accuracy dropped to $59 \%$ on the ambiguous 4 -s trials $(S D=.19)$. Finally, an independent-samples $t$-test on the number of sessions needed to reach criterion of at least $75 \%$ correct in two consecutive sessions in Phase 3 revealed that training

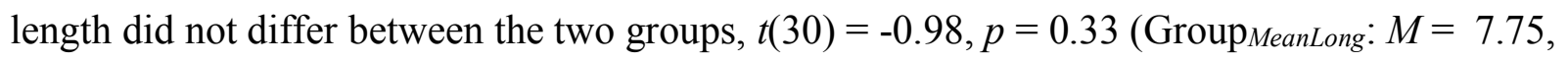
$S D=1.53 ;$ Group $_{\text {MeanShort: }} M=9.31 ; S D=4.25$ sessions).

\section{Discussion}

As in the Upshift Experiment, we again found evidence for the use of both absolute and relative representations in a temporal discrimination task. Our results from the first transfer test (Phase 2) indicated that preserving the absolute and/or relative relations of durations and the associated response locations helped with initial discrimination performance. Furthermore, preserving relative mapping also led to faster adaptation to new temporal and spatial mappings. On the other hand, preventing animals from relying on such relative relations, as in the case of the absolute group, or on both relative and absolute relations, as in the case of the reversal group, resulted in a significant increase in the difficulty of achieving sustained accurate performance in the transfer phase. Furthermore, when we tested the subjects with two novel durations and preserved the relative mapping in Phase 3, we found that the discrimination accuracy showed only slight deterioration, despite the use of an ambiguous duration that was at the arithmetic 
mean of a previously learned duration pair. Although there were several subjects whose discrimination performance deteriorated for the ambiguous cue duration, group accuracy levels were still above chance. Taken together, these findings indicate that animals have knowledge about absolute durations and use them to guide behavior, but they also have knowledge of the relative relationship between durations and this knowledge is not easily modified.

\section{General Discussion}

In the present study, we investigated the nature of temporal encoding in a duration discrimination task. We were particularly interested in understanding whether animals use absolute or relative representations of time to guide their temporal choices. To this end, we trained mice to correctly discriminate different time intervals and then either increased or decreased the cue durations and/or switched the associated response locations, which resulted in changes in relative and/or absolute mappings of duration-lever assignments. Specifically, in the absolute groups, the response that earned reward for one of the durations was preserved from the initial training to the transfer phase. In this group, the subjects had to learn new responses based on the new relative durations. Thus, we would expect positive transfer in this group based on absolute durations and negative transfer based on relative duration. We expected the opposite to be true in the relative groups. These subjects had to learn to make a new response to the duration that was preserved across phases, but the correct action based on relative durations was

preserved. Here, the use of absolute times would lead to negative transfer, but the use of relative times would produce positive transfer. In the reversal groups, subjects had to learn to make new responses based on both absolute and relative representations, and negative transfer should predominate. Overall, our findings indicate that the transfer occurred most readily when relative relationships of durations and response locations were maintained. In contrast, when the animals 
had to re-map these relative relations, their temporal discrimination ability was impaired, and they required extensive training to re-establish temporal control. However, preserving the response location of one of the absolute cue durations in such conditions enabled animals to initially maintain accurate post-shift duration categorization performance. These results demonstrate that animals encode both absolute and relative representations of duration.

At first glance, our findings might seem at odds with previous studies providing evidence for mainly one type of time encoding in humans and nonhuman animals (e.g., Carvalho \& Machado, 2012; Tomaschke et al., 2014; Zentall et al., 2004). However, we think that our evidence for the use of both types of temporal representations is not surprising, as it is wellestablished that organisms can process, learn, and adapt to varying temporal and stimulus contexts (e.g., Akdoğan \& Balc1, 2016; Avlar et al., 2015; De Corte et al., 2018; Jazayeri \& Shadlen, 2010). Even though our findings indicate that categorizations heavily rely on relational control of timing behavior, they also provide clear evidence that animals also utilize absolute temporal information.

Before going into a more detailed discussion on temporal representations, we would like to note that in the absolute and reversal groups, there was a trend for accurate performance to emerge faster to the longer cue than the shorter one. This is, of course, not surprising for the Downshift absolute group that had to press the same lever after the long $(6 \mathrm{~s})$ tone presentations in both phases. However, this finding for the Upshift absolute group is particularly puzzling, as the response location of the short cue (6 s; former long cue) was maintained, which initially produced positive transfer, leading us to expect that the short cue might be learned faster throughout the transfer phase. It is unclear to us whether training history plays a role in learning speeds. More interestingly, why this asymmetry would also be true in the reversal groups is not 
obvious. One possibility is that larger magnitudes may be more salient than smaller ones in general. In fact, a small but diverse body of evidence has shown that discriminations based on odor, sound, physical length, and temporal durations are acquired faster when the outcome is signaled with the larger of the two magnitudes (for a review see Inman \& Pearce, 2018). Although most timing research focuses on steady-state timing behavior and overlooks how temporal control over behavior is acquired (but see Balci et al., 2009; Machado, 1997), however, Church and Deluty (1977) did not report any differences in the acquisition of short vs. long duration discriminations in their seminal temporal bisection study. Therefore, we think that the question of whether the asymmetry appears during the initial training phase or is specific to reversal learning is an interesting one but not settled.

\section{Absolute durations control initial transfer}

In all transfer conditions in all of our experiments the initial transfer seems to be guided by the absolute time durations. In Experiment 1, subjects were first trained on a 2-s vs. 6-s discrimination. When switched to a 6-s vs. 18-s discrimination, the absolute group showed initial positive transfer to the 6-s cue, while the relative and reversal groups showed initial negative transfer. This pattern of results is consistent with the hypothesis that subjects learned to press a particular lever in the presence of the 6-s duration, and initial transfer depended on whether this mapping was preserved. Similarly, in Experiment 2, subjects were first trained on a 6-s vs. 18-s discrimination. When switched to a 2-s vs. 6-s discrimination, the absolute group showed initial positive transfer to the 6-s cue, while the relative and reversal groups showed initial negative transfer. This pattern of is also consistent with the hypothesis that subjects learned to press a particular lever in the presence of the 6-s duration, and initial transfer depended on whether this mapping was preserved. In the last phase of Experiment 2 (Phase 3), subjects were transferred to 
a 4-s vs. 12-s discrimination. These values were the arithmetic means of the duration pairs used in Phase 2. Specifically, the 12-s cue was the arithmetic mean of the 6- and 18-s cue durations learned by the control and reversal mice, and the 4-s cue was the arithmetic mean of the 2- and 6$\mathrm{s}$ cues that were used for the relative and absolute mice. The relative mapping was the same as in the previous phase for all groups. Based on generalization from the absolute durations of Phase 2, we expected that the duration that was at the mean of the previous durations to neither help nor hinder the transfer, and that the novel cue to be facilitated. For example, in the groups transferred from the 6-s vs. 18-s discrimination we expected that the tendency to press the former 6-s lever would generalize strongly to a 4-s cue, and there would be an equal tendency to press both levers after the 12-s cue. This is indeed what we found in the first session. Similarly, in the groups transferred from the 2-s vs. 6-s discrimination, we expected that the tendency to press former 6-s lever would generalize strongly to a 12 -s cue and there would be an equal tendency to press both levers after the 4-s cue. This pattern too was confirmed in the first session data for these groups. Overall, our data provide strong evidence that the dominant representation that governs performance at the start of a transfer test is an absolute one. Clearly, the relation between absolute durations and specific responses is encoded during temporal discriminations.

\section{Relative representations exert long lasting control over responding}

Several features of the data suggest that relative representations of durations are also encoded and used in temporal discrimination learning. In both experiments, there was initially positive transfer to the preserved duration in the absolute transfer groups. In these groups, subjects had to learn to make new responses based on relative durations. Surprisingly, despite the positive initial transfer, performance to the preserved duration deteriorated over the first few sessions of training and most subjects never recovered baseline accuracy. 
One explanation for this decline in discrimination performance to the preserved duration is that the transfer test temporarily reduced stimulus control by cue durations and strengthened stimulus control by lever locations (Carvalho \& Machado, 2012; Carvalho et al., 2016). Take the case of the Downshift absolute group: After learning to press the left lever following the 6-s cue and the right lever following the 18-s cue, the animals learned to press the right lever following the 2-s cue and continued to press the left lever following the 6-s cue. Due to temporal generalization, most of these mice started to choose the left lever after the 2-s cue and experienced extinction for pressing the left lever. The occasional reinforcements following presses on the right lever as well as the extinction episodes could explain the generalized bias towards the right lever, and thus the marked decline in discrimination accuracy on 6-s trials. This competition between stimulus control by cue durations and lever locations could explain the acquisition trade-off observed in most of the transfer sessions in the absolute groups. If this were the explanation for the deterioration in performance, then we should see identical changes in the Downshift relative group. After learning to press the left lever following the 6-s cue and the right lever following the 18-s cue, the animals learned to press the left lever following the 2-s cue and the right lever following the 6-s cue. Due to temporal generalization, most of these mice started to choose the left lever after both 2- and 6-s cues. As training continues for the 6-s cue, the reinforcements following presses on the right lever as well as the extinction on the left lever should strengthen the tendency to make right lever responses. Generalization of this tendency to the 2-s cue should produce a deterioration of performance on the left lever as it did in the absolute group. Instead, despite initially showing negative transfer to the preserved duration, subjects in the relative groups of both experiments recovered high accuracy very rapidly without any deterioration in performance. Based on generalization alone we would not expect a 
difference between absolute and relative groups. We, therefore, think that relative representations also play a particularly crucial role when animals are learning a new duration pair.

Taken together, these patterns of results suggest that animals might use single or multiple types of temporal representations to learn task contingencies, which might change during the course of training or when tested under different experimental conditions. One possibility is that subjects had learned to respond on the basis of absolute durations in Phase 1, but the relative relation between durations guided the remapping of responses. It is also possible that, subjects learn temporal discriminations with relative representations and with training they switch to using absolute representations. When the absolute representations fail to work in the transfer phase, perhaps they then revert to using relative representations. Alternatively, perhaps they initially learn with absolute representations and only switch to relative ones when the first strategy fails. It is not clear why one type of representation would be favored over the other one in this task, as both could result in successful performance.

The third phase of Experiment 2 is consistent with these speculations. In that phase, one of the durations was the mean of the durations experienced in the previous phase. This meant that based on control by absolute time the two choices ought to be of approximately equal strength. Furthermore, in this test the relative mapping of durations to responses was preserved. Here, where there was no basis for negative or positive transfer from an absolute encoding perspective, we observed positive transfer in many subjects and rapid acquisition of the new discrimination, as would be expected from having preserved the relative mapping.

\section{Metric representations of time}

Even though our study shows that animals form both absolute and relative temporal representations, one particularly interesting finding was the amount of individual differences in 
how fast the animals adapted to the uncertainty owing to the changes in temporal and spatial contingencies. Our statistical test results as well as the visual inspection of individual subjects' data consistently revealed that some animals showed immediate transfer, whereas others required extensive training to learn new task parameters, even when tested under the same experimental conditions. These findings collectively raise the questions of at what point mice learn relative and absolute time representations and to what extent they utilize either type of temporal information to guide their time judgments in stable and dynamic task structures. It is possible that animals mainly rely on one type of representation in a stable temporal context and then flexibly adjust the type of temporal code after facing more uncertain environments in which there is variability in the number and the length of time intervals, as well as in the associated responses. However, it is a methodological challenge to examine such "subjectivity" of temporal representations and response strategies, as it is unclear when individual animals detect or perceive changes in temporal contexts and update their beliefs accordingly. Nonetheless, these outstanding questions about how time is represented under stable and uncertain conditions should be addressed at both the behavioral and the neural levels to provide a more thorough understanding of the nature of temporal codes.

As a way of understanding the different ways in which time can guide behavior, we suggest that the underlying representation of time has the properties of a ratio scale. Consequently, when asked to discriminate between two durations, one can use this metric knowledge in multiple ways. Subjects could learn to respond in the presence of a specific duration. This is what we have referred to as the absolute representation. Alternatively, via subtraction or addition from a standard in memory, subjects can respond to the larger of two magnitudes. Alternatively, via division the larger or smaller alternative can be determined. The 
latter two computations are what we have referred to as the relative representations. While we can conclude that absolute and relative representations were used in the current experiments, we cannot say which of the possible computations underlie the formation of relative representations.

Relevantly, there is mounting evidence that there are reliable neural correlates of a metric representation of time that suggest that both absolute and relative relationships are computed. For instance, Mello et al. (2015) recorded from individual striatal neurons in rats during a serial fixed-interval task in which they varied the length of fixed time intervals in different test blocks, after which food rewards became available. Their results showed that at the population level, two-thirds of striatal neurons exhibited a similar temporal trajectory in firing patterns and maintained their ordinal peak activity position across different target intervals ranging from 12$60 \mathrm{~s}$. The rescaling of the firing patterns with changes in duration, provides support mainly for relative encoding of time in the striatum. A particularly relevant study by Shimbo et al. (2021) recently investigated the hippocampal CA1 neuronal activity in rats during a duration discrimination task, in which duration pairs were scaled up or down in different blocks of trials (e.g., block 1: “10s/Left, 5s/Right”; block 2: "20s/Left, 10s/Right”; block 3: "10s/Left, 5s/Right”. Their findings revealed that activity patterns of an assembly of CA1 pyramidal cells compressed or stretched in time as a function of the increases or decreases in the durations of timed intervals, suggesting scalable temporal representations in the rat hippocampus.

Such compression or dilation observed in neural activity in response to changes in temporal contingencies, referred to as temporal scaling, has been reported also in other brain regions and behavioral tasks (e.g., Emmons et al., 2017; Gouvêa et al., 2015; Komura et al., 2001; Leon \& Shadlen, 2003; Wang et al., 2018; Xu et al., 2014). However, despite the clear evidence for such relative neural signals in the brain, which are potentially useful for adaptive 
behavior and efficient neural processing, Mello et al. (2015) also reported that one-third of neurons in the striatum did not preserve their ordinal position in their firing activity across different target durations. Perhaps these neurons were encoding absolute temporal information which could be multiplexed in striatal neuron populations (Motanis \& Buonomano, 2015). Previous research on the neural basis of temporal encoding also provided evidence consistent with the existence of temporally specific neurons in the striatum by demonstrating that neurons show distinct firing patterns for individual durations (Matell et al., 2003). Furthermore, other studies show that only a subset of neurons involved in timing rescaled (e.g., Meirhaeghe et al., 2021; Mello et al., 2015; Shimbo et al., 2021), which indicates that a proportion of neurons possibly also encodes absolute temporal information (Motanis \& Buonomano, 2015).

Here it is important to note that, most previous studies on the neural basis of temporal processing assume that an absolute temporal representation takes the form of neural activity responding to specific durations, whereas relative temporal representations refer to the adaptive rescaling of neural activity to different time intervals. Based on our study as well as other behavioral findings mainly from the Machado lab, we would like to caution the reader against this dichotomy and suggest that time is represented in a way that allows for multiple representations to be computed to guide performance.

In our experiments we pitted the different types of representations against one another in the transfer tests. It appears that while only one type of temporal representation dominates at different points of training, multiple representations may exist. In the absolute groups, there was an initial positive transfer based on retraining the same relationship between an absolute time and a specific response. However, as training progressed it appeared the subjects became aware that the preserved duration, though of the same absolute duration, was no longer in the same relative 
position (longer or shorter). This conflict produced a very pronounced deterioration of performance that only slowly recovered with extensive training. Whether this strong control by the relative representation is a general principle or specific to the current task and training procedure is worthy of future study, as task structures might contribute to the variability observed in how the brain encodes time (Zhou et al., 2022). Another important aspect of understanding the neural basis of timing and our findings will be to understand how time-varying patterns of neural activity (e.g., population clocks, ramping activity, etc.) are mapped onto motor outputs during learning.

In sum, the current set of experiments show that mice can represent experienced durations both as having a certain magnitude (absolute representation) and as being shorter or longer of the two durations (an ordinal relation to other cue durations), with relational control having an enduring influence in governing temporal discriminations. We think that it is actually this ability of describing temporal relations and event durations in various ways that enables organisms to perform arithmetic operations of time intervals in an effective manner. Consequently, individuals are able to make common relativized descriptions such as identifying the shortest and or the longest durations, or computing the average of different time intervals in various contexts to adapt behavior and guide decisions. It also enables brains to compute the sum or difference of a number of durations (Akdoğan et al., unpublished; Gibbon \& Church, 1981; Takahashi \& Watanabe, 2015; Wearden, 2002), which helps individuals to adaptively execute behaviors at the correct time and in the correct amount of time. 


\section{References}

Akdoğan, B., \& Balc1, F. (2016). Stimulus probability effects on temporal bisection performance of mice (Mus musculus). Animal Cognition, 19, 15-30. https://doi.org/10.1007/s10071$\underline{015-0909-6}$

Avlar, B., Kahn, J. B., Jensen, G., Kandel, E. R., Simpson, E. H., \& Balsam, P. D. (2015). Improving temporal cognition by enhancing motivation. Behavioral Neuroscience, 129(5), 576. https://doi.org/10.1037/bne0000083

Balci, F., Gallistel, C. R., Allen, B. D., Frank, K. M., Gibson, J. M., \& Brunner, D. (2009). Acquisition of peak responding: What is learned?. Behavioural Processes, 80(1), 67-75. https://doi.org/10.1016/j.beproc.2008.09.010

Balsam, P. D, Fairhurst, S., \& Gallistel, C. R. (2006). Pavlovian contingencies and temporal information. Journal of Experimental Psychology: Animal Behavior Processes, 32, 28494. https://doi.org/10.1037/0097-7403.32.3.284

Balsam, P. D, \& Gallistel, C. R. (2009). Temporal maps and informativeness in associative learning. Trends in Neurosciences, 32, 73-78. https://doi.org/10.1016/j.tins.2008.10.004

Brannon, E. M., \& Terrace, H. S. (1998). Ordering of the numerosities 1 to 9 by monkeys. Science, 282, 746-749. https://doi.org/10.1126/science.282.5389.746

Brannon, E. M., \& Terrace, H. S. (2000). Representation of the numerosities 1-9 by rhesus macaques (Macaca mulatta). Journal of Experimental Psychology: Animal Behavior Processes. 26, 31-49. https://doi.org/10.1037/0097-7403.26.1.31

Carvalho, M. P., \& Machado, A. (2012). Relative versus absolute stimulus control in the temporal bisection task. Journal of the Experimental Analysis of Behavior, 98(1), 23-44. https://doi.org/10.1901/jeab.2012.98-23 
Carvalho, M. P., Machado, A., \& Tonneau, F. (2016). Learning in the temporal bisection task: Relative or absolute?.Journal of Experimental Psychology: Animal Learning and Cognition, 42(1), 67. https://doi.org/10.1037/xan0000089

Church, R. M. (1984). Properties of the internal clock. Annals of the New York Academy of Sciences, 423, 566-582. https://doi.org/10.1111/j.1749-6632.1984.tb23459.x

Church, R. M., \& Deluty, M. Z. (1977). Bisection of temporal intervals. Journal of Experimental Psychology: Animal Behavior Processes, 3(3), 216. https://doi.org/10.1037/00977403.3.3.216

Davis, H. (1984). Discrimination of the number three by a raccoon (Procyon lotor). Animal Learning \& Behavior, 12(4), 409-413. https://doi.org/10.3758/BF03199987

De Corte, B. J., Della Valle, R. R., \& Matell, M. S. (2018). Recalibrating timing behavior via expected covariance between temporal cues. eLife, 7:e38790. https://doi.org/10.7554/eLife.38790.001

Emmons, E. B., De Corte, B. J., Kim, Y., Parker, K. L., Matell, M. S., \& Narayanan, N. S. (2017). Rodent medial frontal control of temporal processing in the dorsomedial striatum. Journal of Neuroscience, 37(36), 8718-8733. https://doi.org/10.1523/JNEUROSCI.1376$\underline{17.2017}$

Fetterman, J. G., Dreyfus, L. R., \& Stubbs, D. A. (1989). Discrimination of duration ratios. Journal of Experimental Psychology: Animal Behavior Processes, 15(3), 253-263. https://doi.org/10.1037/0097-7403.15.3.253

Fetterman, J. G., Dreyfus, L. R., \& Stubbs, D. A. (1993). Discrimination of duration ratios by pigeons (Columba livia) and humans (Homo sapiens). Journal of Comparative Psychology, 107(1), 3-11. https://doi.org/10.1037/0735-7036.107.1.3 
Gershman, S. J., \& Uchida, N. (2019). Believing in dopamine. Nature Reviews Neuroscience, 20, 703-714. https://doi.org/10.1038/s41583-019-0220-7

Gibbon, J. (1977). Scalar expectancy theory and Weber's Law in animal timing. Psychological Review, 84, 279-335. https://doi.org/10.1037/0033-295X.84.3.279

Gibbon, J., \& Church, R. M. (1981). Time left: Linear versus logarithmic subjective time. Journal of Experimental Psychology: Animal Behavior Processes, 7, 87-108. https://doi.org/10.1037/0097-7403.7.2.87

Gibbon, J., Church, R. M., \& Meck, W. H. (1984). Scalar timing in memory. In J. Gibbon \& L. Allan (Eds.), Timing and time perception (Vol. 423, pp. 52-77). New York Academy of Sciences.

Gouvêa, T.S., Monteiro, T., Motiwala, A., Soares, S., Machens, C., \& Paton, J. J. (2015). Striatal dynamics explain duration judgments. eLife, 4:e11386.

https://doi.org/10.7554/eLife.11386

Hardy, N., \& Buonomano, D.V. (2018). Encoding time in feedforward trajectories of a recurrent neural network model. Neural Computation, 30, 378-396. https://doi.org/10.1162/neco_a 01041

Inman, R. A., \& Pearce, J. M. (2018). The discrimination of magnitude: A review and theoretical analysis. Neurobiology of Learning and Memory, 153(Pt B), 118-130. https://doi.org/10.1016/j.nlm.2018.03.020

Jazayeri, M., \& Shadlen, M. N. (2010). Temporal context calibrates interval timing. Nature Neuroscience, 13(8), 1020. https://doi.org/10.1038/nn.2590 
Kamil, A. C., \& Jones, J. E. (2000). Geometric rule learning by Clark's nutcrackers (Nucifraga columbiana). Journal of Experimental Psychology: Animal Behavior Processes, 26(4), 439. https://doi.org/10.1037/0097-7403.26.4.439

Karmarkar, U. R., \& Buonomano, D. V. (2007). Telling time in the absence of clocks. Neuron, 53(3), 427-438. https://doi.org/10.1016/j.neuron.2007.01.006

Komura, Y., Tamura, R., Uwano, T., Nishijo, H., Kaga, K., \& Ono, T. (2001). Retrospective and prospective coding for predicted reward in the sensory thalamus. Nature, 412, 546-549. https://doi.org/10.1038/35087595

Leon, M. I., \& Shadlen, M. N. (2003). Representation of time by neurons in the posterior parietal cortex of the macaque. Neuron, 38, 317-327. https://doi.org/10.1016/S0896$\underline{6273(03) 00185-5}$

Machado A. (1997). Learning the temporal dynamics of behavior. Psychological Review, 104(2), 241-265. https://doi.org/10.1037/0033-295x.104.2.241

Maia, S., \& Machado, A. (2009). Representation of time intervals in a double bisection task: relative or absolute? Behavioural Processes, 81, 280-285. https://doi.org/10.1016/j.beproc.2008.10.012

Matell, M. S., Meck, W. H., \& Nicolelis, M. A. (2003). Interval timing and the encoding of signal duration by ensembles of cortical and striatal neurons. Behavioral Neuroscience, 117(4), 760-773. https://doi.org/10.1037/0735-7044.117.4.760

Meck, W. H., \& Church, R. M. (1983). A mode control model of counting and timing processes. Journal of Experimental Psychology: Animal Behavior Processes, 9(3), 320. https://doi.org/10.1037/0097-7403.9.3.320 
Meirhaeghe, N., Sohn, H., \& Jazayeri, M. (2021). A precise and adaptive neural mechanism for predictive temporal processing in the frontal cortex. bioRxiv. https://doi.org/10.1101/2021.03.10.434831

Mello, G. B. M., Soares, S., \& Paton, J. J. (2015). A scalable population code for time in the striatum. Current Biology, 25, 1113-1122. https://doi.org/10.1016/j.cub.2015.02.036

Molet, M., \& Zentall, T. R. (2008). Relative judgments affect assessments of stimulus duration. Psychonomic Bulletin \& Review, 15(2), 431-436. https://doi.org/10.3758/BF03211789

Motanis, H., \& Buonomano, D. V. (2015). Neural coding: Time contraction and dilation in the striatum. Current Biology, 25, R374-R376. https://doi.org/10.1016/j.cub.2015.02.057

Petter, E.A., Gershman, S. J., \& Meck, W. H. (2018). Integrating models of interval timing and reinforcement learning. Trends in Cognitive Science, 22(10), 911-922. https://doi.org/10.1016/j.tics.2018.08.004

Russell, R., \& Kirkpatrick, K. (2007). The role of temporal generalization in a temporal discrimination task. Behavioural Processes, 74(2), 115-125. https://doi.org/10.1016/j.beproc.2006.08.004

Shimbo, A., Izawa, E. I., \& Fujisawa, S. (2021). Scalable representation of time in the hippocampus. Science Advances, 7(6), eabd7013. https://doi.org/10.1126/sciadv.abd7013

Spence, K. W. (1938). Gradual versus sudden solution of discrimination problems by chimpanzees. Journal of Comparative Psychology, 25(1), 213224. https://doi.org/10.1037/h0063375

Spetch, M. L., Rust, T. B., Kamil, A. C., \& Jones, J. E. (2003). Searching by rules: Pigeons' (Columba livia) landmark-based search according to constant bearing or constant 
distance. Journal of Comparative Psychology, 117, 123-132. https://doi.org/10.1037/0735-7036.117.2.123

Spínola, I., Machado, A., de Carvalho, M. P., \& Tonneau, F. (2013). What do humans learn in a double, temporal bisection task: Absolute or relative stimulus durations? Behavioural Processes, 95, 40-49. https://doi.org/10.1016/j.beproc.2013.01.003

Stevens, S. S. (1946). On the theory of scales of measurement. Science, 103(2684), 677-680.

Takahashi, K. \& Watanabe, K. (2015). Mental summation of temporal duration within and across senses. PLoS ONE, 10: e0141466. https://doi.org/10.1371/journal.pone.0141466

Taniuchi, T., Sugihara, J., Wakashima, M., \& Kamijo, M. (2016). Abstract numerical discrimination learning in rats. Learning \& Behavior, 44(2), 122-136. https://doi.org/10.3758/s13420-016-0209-2

Walsh, V. (2003). A theory of magnitude: common cortical metrics of time, space and quantity. Trends in Cognitive Sciences, 7(11), 483-488. https://doi.org/10.1016/j.tics.2003.09.002

Wan, M., Djourthe, M., Taylor, K. M., \& Balsam, P. D. (2010). Relative temporal representations in Pavlovian conditioning. Behavioural Processes, 83(2), 154-161. https://doi.org/10.1016/j.beproc.2009.11.012

Wang, J., Narain, D., Hosseini, E. A., \& Jazayeri, M. (2018). Flexible timing by temporal scaling of cortical responses. Nature Neuroscience, 21, 102-110. https://doi.org/10.1038/s41593$\underline{017-0028-6}$

Ward, R. D., Kellendonk, C., Simpson, E. H., Lipatova, O., Drew, M. R., Fairhurst, S., ... \& Balsam, P. D. (2009). Impaired timing precision produced by striatal D2 receptor overexpression is mediated by cognitive and motivational deficits. Behavioral Neuroscience, 123(4), 720-730. https://doi.org/10.1037/a0016503 
Wearden, J. H. (2002). Traveling in time: A time-left analogue for humans. Journal of Experimental Psychology: Animal Behavior Processes, 28, 200-208. https://doi.org/10.1037//0097-7403.28.2.200

Xu, M., Zhang, S,-y., Dan, Y., \& Poo, M.-m. (2014). Representation of interval timing by temporally scalable firing patterns in rat prefrontal cortex. Proceedings of the Natural Academy of Sciences USA, 111, 480-485. https://doi.org/10.1073/pnas.1321314111

Zentall, T. R., Weaver, J. E., \& Clement, T. S. (2004). Pigeons group time intervals according to their relative duration. Psychonomic Bulletin \& Review, 11(1), 113-117. https://doi.org/10.3758/BF03206469

Zhou, S., Masmanidis, S. C., \& Buonomano, D. V. (2022). Encoding time in neural dynamic regimes with distinct computational tradeoffs. PLOS Computational Biology, 18(3), e1009271. https://doi.org/10.1371/journal.pcbi.1009271 


\section{Supplemental Online Material}

Journal of Experimental Psychology: Animal Learning and Cognition

Temporal Encoding: Relative and Absolute Representations of Time Guide Behavior

Başak Akdoğan*, Amita Wanar, Benjamin K. Gersten, Charles R. Gallistel, and Peter D Balsam

*Corresponding Author

Başak Akdoğan, M.A.

Department of Psychology

Columbia University

New York, NY 10027, USA

Email: basak.akdogan@columbia.edu 

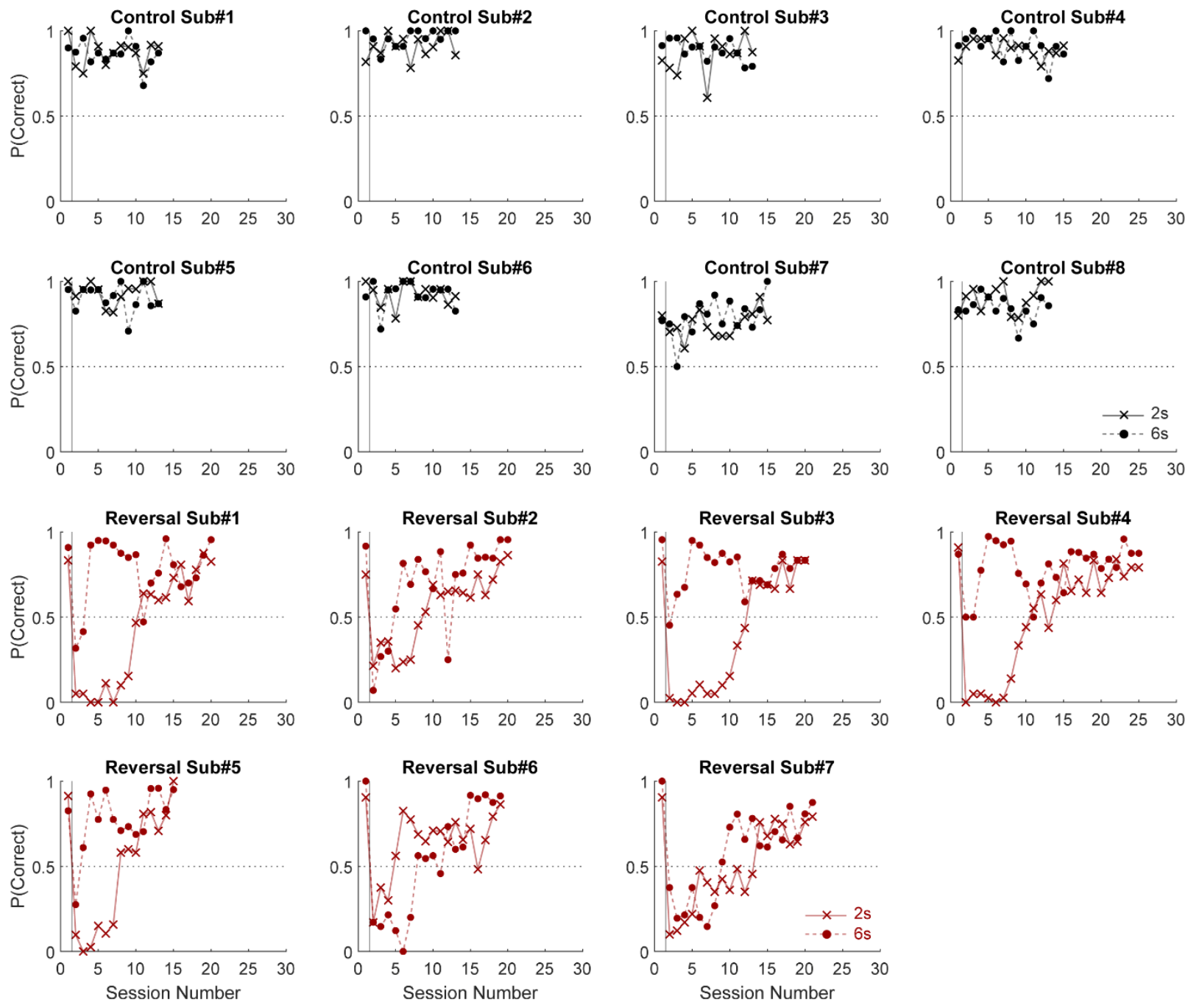

Figure S1. Individual acquisition curves of the control and reversal subjects in Experiment 1. Vertical lines indicate the start of the transfer test. The proportion correct of responses on short and long trials in both the last pre-transfer session and all post-transfer sessions are shown for each individual animal. 

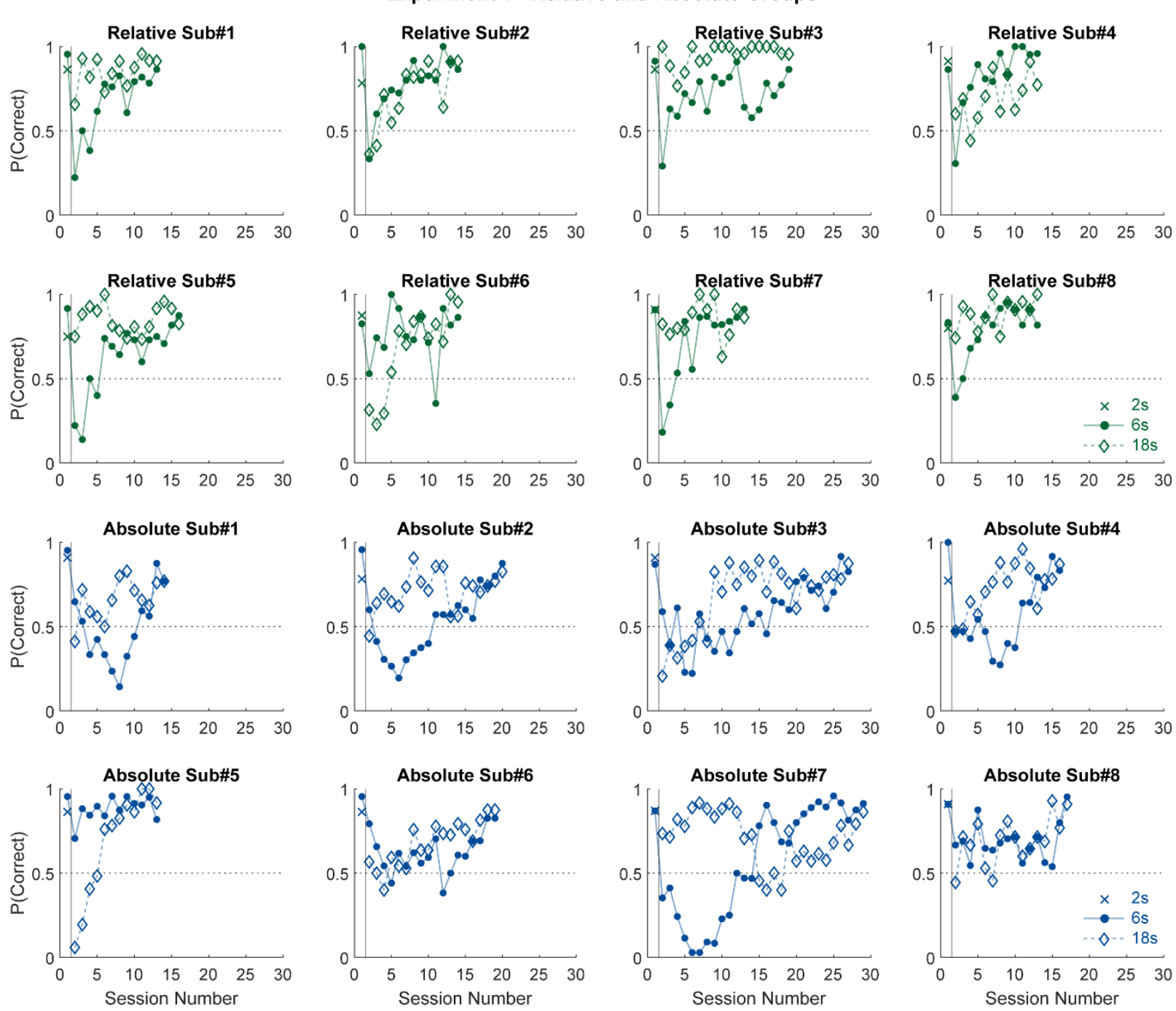

Figure S2. Individual acquisition curves of the relative and absolute subjects in Experiment 1. Vertical lines indicate the start of the transfer test. The proportion correct of responses on short and long trials in both the last pre-transfer session and all post-transfer sessions are shown for each individual animal. 

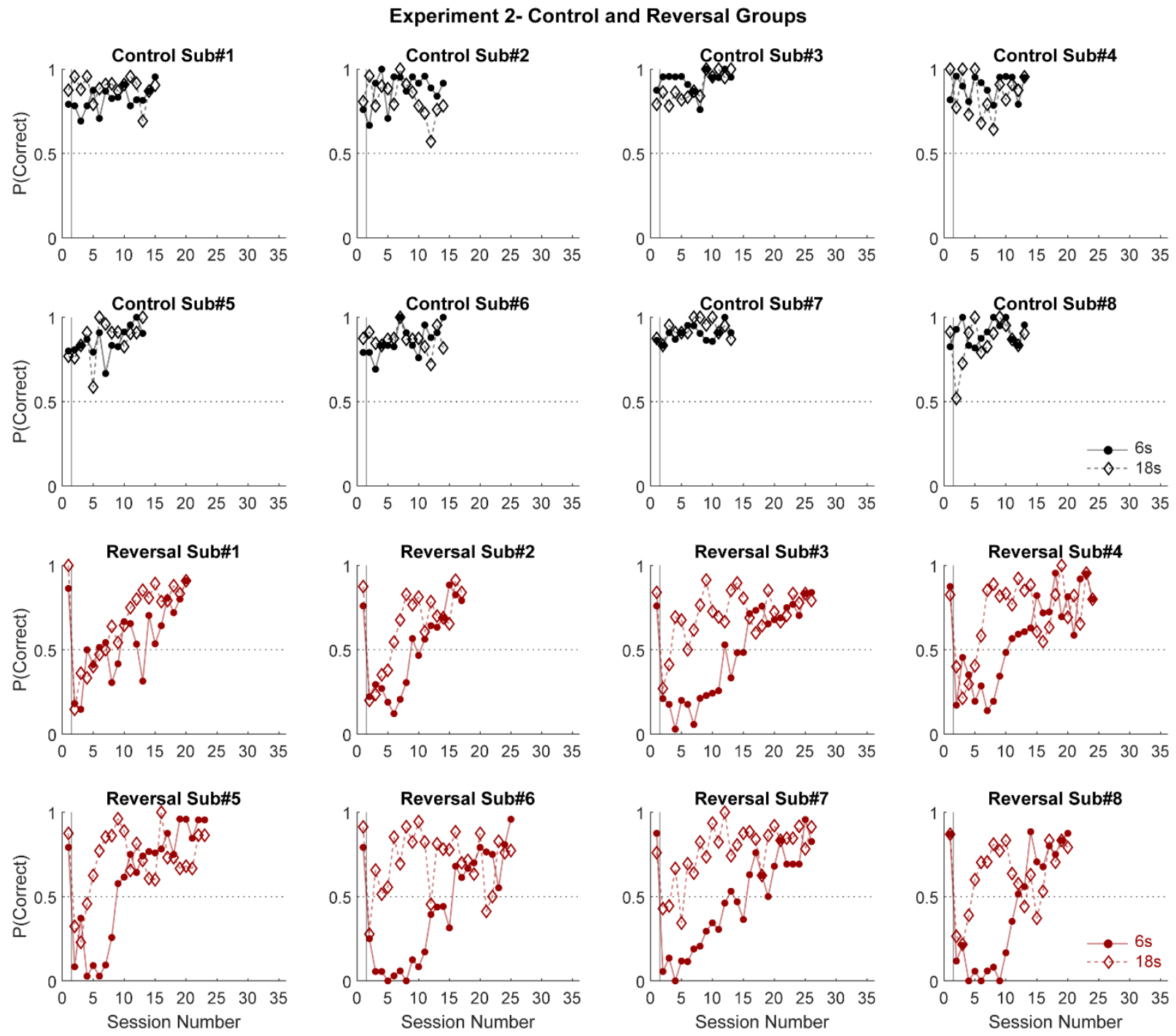

Figure S3. Individual acquisition curves of the control and reversal subjects in Experiment 2. Vertical lines indicate the start of the transfer test. The proportion correct of responses on short and long trials in both the last pre-transfer session and all post-transfer sessions of Phase 2 are shown for each individual animal. 

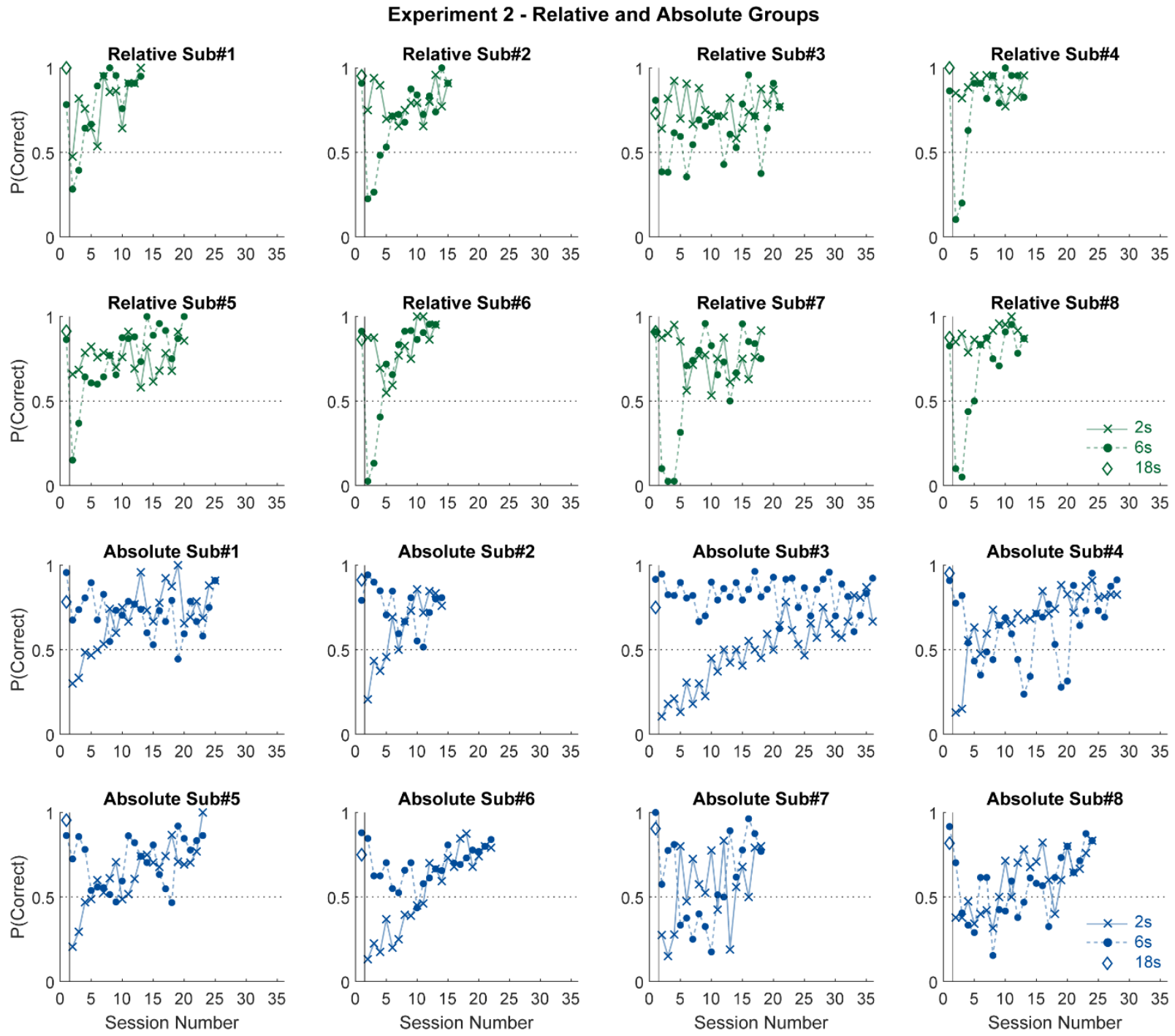

Figure S4. Individual acquisition curves of the relative and absolute subjects in Experiment 2. Vertical lines indicate the start of the transfer test. The proportion correct of responses on short and long trials in both the last pre-transfer session and all post-transfer sessions of Phase 2 are shown for each individual animal. 